\title{
Forecasting and Turning Point Predictions in a Bayesian Panel VAR Model
}

\author{
Fabio Canova* \\ Universitat Pompeu Fabra, University of Southampton and CEPR \\ and \\ Matteo Ciccarelli ${ }^{\dagger}$ \\ Universitat d'Alacant
}

This Draft: October 1999

\begin{abstract}
A bstract
We provide methods for forecasting variables and predicting turning points in pane Bayesian VARs. Wespecify $a^{\circ}$ exible model which accounts for both interdependencies in the cross section and time variations in the parameters. Posterior distributions for the parameters are obtained for a particular type of di @ise, for Minnesota-type and for hierarchical priors. Formulas for multistep, multiunit point and average forecasts are provided. An application to the problem of forecasting the growth rate of output and of predicting turning points in the G-7 illustrates the approach. A comparison with alternative forecasting methods is also provided.
\end{abstract}

Key Words: Forecasting, Turning Points, Bayesian Methods, Panel VAR, Markov Chains Monte Carlo Methods

J el Classi' ${ }^{-}$cation nos: C11, C15, E32, E37

\footnotetext{
*We would like to thank Albert Marcet and the participants of the 1999 European Meetings of the Econometric Society for comments and suggestions.

${ }^{\dagger}$ Canova, Departament d'Economia i Empresa, UPF, Ramon Trias Fargas 25-27, 08005 Barcelona, Spain. E-mail: canova@upf.es; Ciccarelli, Fundamentos del Análisis Económico, UA, Ap. Correos 99, 03080 Alicante, Spain.
} 


\section{Introduction}

Pane VAR models have become increasingly popular in macroeconomics to study the transmission of shodks across countries (Ballabriga, Sebastian and Valles (1995)), the propagation erects of monetary policy in the European Union (Gerlach and Smets (1996)) and the average di Berential response of developed and underdeveloped countries to domestic and external disturbances (Ho ${ }^{\circledR}$ maister and Rold\$s (1997), Rebucci (1998)). At the same time, recent developments in computer technology have permitted the estimation of increasingly complex multicountry VAR models in reasonable time, making them potentially usable for a variety of forecasting and policy purposes.

Despite this interest, the theory for panel VAR is somewhat underdeveloped. After the works of Chamberlain (1982, 1984) and Holtz\{Eakin et al. (1988), who specify panel VAR models for micro data, to the best of our knowledge only Pesaran and Smith (1996), Canova and Marcet (1997) and Hsiao et al. (1998) have considered problems connected with the speci ${ }^{-}$cation and the estimation of (univariate) dynamic macro panels. Garcia Ferrer et al. (1987), Zellner and Hong (1989), Zellner, Hong and Min (1991), on the other hand, have provided Bayesian shrinkage estimators and predictors for similar models. In general, a researcher focuses on the speci ${ }^{-}$cation

$$
\mathrm{y}_{i t}=\mathrm{A}(\mathrm{L}) \mathrm{y}_{i t-1}+{ }{ }_{i t}
$$

where $\mathrm{y}_{i t}$ is a $\mathrm{G}$ \{dimensional vector, $\mathrm{i}=1 ;:: ; \mathrm{N}$; $\mathrm{A}(\mathrm{L})$ is a matrix in the lag operator; " ${ }_{i t}=$

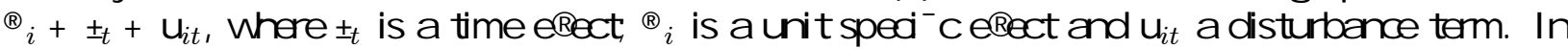
some cases (see e.g. Holtz\{Eakin et al. (1988)) a speci ${ }^{-}$cation with time varying slope coe \pm cients and $a^{-}$xed erect is used. Two main restrictions characterize this speci ${ }^{-}$cation. First, it assumes common slope coe \pm cients. Second, it does not allow for interdependencies across units. With these restrictions, the interest is typically in estimating the average dynamics of the system in response to shocks (the matrix $A(L)$ ).

Garcia Ferrer et al., Canova and Marcet and Pesaran and Smith, instead, use a univariate dynamic model of the form

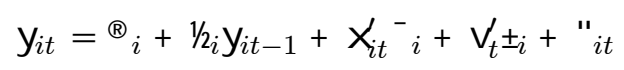

where $y_{i t}$ is a scalar, $x_{i t}$ is a set of $k$ exogenous unit speci ${ }^{-} c$ regressors, $v_{t}$ is a set of $h$ exogenous regressors common to all units while ${ }^{1 / z},{ }_{i}{ }_{i}$ and $\pm_{i}$ are unit speci ${ }^{-} \mathrm{c}$ vectors of coe + cients. In some speci ${ }^{-}$cations these vectors of coe \pm cients are assumed to have an exchangeable prior. Two restrictions are implicit also in this speci ${ }^{-}$cation. First, no time variation is allowed in the parameters. Second, there are no interdependencies either among di Berent variables within units or among the same variable across units.

The task of this paper is to relax these restrictions and study the issues of speci ${ }^{-}$cation, estimation and forecasting in a macro-panel VAR model with interdependencies. Our point of view is Bayesian. Such an approach has been widely used in the VAR literature since the works of Doan, Litterman and Sims (1984), Litterman (1986), and Sims and Zha (1998) and provides a convenient framework where one can allow for both interdependencies and meaningful time variations in the coe \pm cients. The speci ${ }^{-}$cation we consider has the general form

$$
\mathrm{y}_{i t}=\mathrm{A}_{i t}(\mathrm{~L}) \mathrm{Y}_{t-1}+{ }{ }_{i t}
$$


where $Y_{s}(s<t)$ is a vector of $G N$ elements (there $G$ variables for each unit $\mathrm{i}=1 ;::: \mathrm{N}$ ). Because coe \pm cients vary across units and along time, estimation of the parameters is impossible without imposing restrictions. However, instead of constraining the coe \pm cients to be the same across units, we assume that they are random and a prior distribution on $A_{i t}(L)$ is introduced. We decompose the parameter vector into two components, one which is unit speci ${ }^{-} \mathrm{c}$ and the other which is time speci ${ }^{-} \mathrm{c}$. We specify a ${ }^{\circ}$ exible prior on these two components which parsimoniously takes into account possible interdependencies in the cross section and allows for time variations in the evolution of the parameters over time The prior shares features with those of Lindlay and Smith (1972), Doan, Litterman and Sims (1984) and Hsiao et al. (1998) and it is speci ed to havea hierarchical structure, which allows for various degrees of ignorance in the researcher's information about the parameters.

Besides important considerations concerning the speci- cation of the model, Bayesian VARs are known produce better forecasts than unrestricted VAR and, in many situations, ARIMA or structural models (Canova (1995) for references). By allowing interdependencies and some degree of information pooling across units we introduce an additional level of ${ }^{\circ}$ exibility which may improve the forecasting ability of these models.

We analyze several special cases of our speci ${ }^{-}$cation and compute Bayesian estimators for the individual coe \pm cients and for their mean values over the cross section. In some cases analytical formulas for the posterior mean are available using standard formulas. Whenever the parameters of the prior are unknown, we employ the predictive density of the model to estimate them and plug-in our estimates in the relevant formulas in an empirical Bayes fashion.

In the case of fully hierarchical priors, a Markov Chain Monte Carlo method (theGibbs sampler) is employed to calculate posterior distributions. Such an approach is particularly useful in our setup since it exploits the recursive features of the posterior distribution. We provide recursive formulas for multistep, multiunit forecasts, consistent with the information available at each point in time using the posterior of the parameters or the predictive density of future observations. The predictive density of future observation is also used to compute turning point probabilities.

To illustrate the forecasting ability of the proposed approach, we apply the methodology to the problem of predicting output growth, of forecasting turning points in output growth and computing the probability of a recession in the G-7 using three variables (output growth, real stock returns and real money growth) for each country in the panel. To evaluate the performance of the model we also provide a forecasting comparison with other speci cations suggested in the literature. We show that our pane VAR approach improves over existing univariate and simple BVAR models when we measure the forecasting performance using the Theil-U and the MAD criteria, both at the one step and at the four steps horizons. The improvements are of the order of $5-10 \%$ with the Theil-U and about 2-4\% with the MAD. The forecasting performance of our speci ${ }^{-}$cation is also slightly better then the one of a BVAR model which mechanically extends the Litterman prior to the panel case In terms of turning point predictions, the two versions of our panel approach are able to recognize about $80 \%$ of turning points in the sample and they turn out to be the best for this task, along with Zellner's g-prior shrinkage approach. The simple extension of the Litterman's prior to the panel case does poorly along this dimension and, among all the procedures employed, is the second worst. Finally, we show that the proposed method is competitive with 
the best speci ${ }^{-}$cations in predicting the peak in US economic activity occurred in 1990:3 when using the information available in 1988:4, a peak which was missed by many of the commercial and government forecasting procedures. Depending on the speci ${ }^{-}$cation, our approach ${ }^{-}$nds $20-55 \%$ probability of a downward turn at that date.

The rest of the paper is organized as follows. The next section gives the general model speci- cation and the assumptions we make Section 3 provides the generalities of Bayesian estimation of the model. Section 4 speci ${ }^{-}$es the prior and discusses the computational issues involved. Section 5 describes formulas for multi-step, multi-units forecasting. Section 6 contains the forecasting application to a panel VAR model for the G-7. Section 7 condudes.

\section{The general specification}

The statistical reduced form mode we use is of the form:

$$
\mathrm{y}_{i t}=\mathrm{X}_{j=1}^{\mathrm{N}^{N} \mathrm{X}^{p}} \mathrm{~b}_{i t, l}^{j} \mathrm{y}_{j t-l}+\mathrm{d}_{i t} \mathrm{v}_{t}+\mathrm{u}_{i t}
$$

where $\mathrm{i}=1 ; \ldots ; \mathrm{N} ; \mathrm{t}=1 ; \ldots ; ; \mathrm{T} ; \mathrm{y}_{i t}$ is a $\mathrm{G}\left\{\right.$ dimensional vector for each $\mathrm{i}, \mathrm{b}_{i t, l}^{j}$ are $\mathrm{G} f \mathrm{G}$ matrices, $\mathrm{d}_{i t}$ is $\mathrm{G} f \mathrm{q} ; \mathrm{v}_{t}$ is a $\mathrm{q} f 1$ vector of exogenous variables common to all units and $\mathrm{u}_{i t}$ is a $\mathrm{G}$ \{dimensional vector of random disturbances. Here $p$ is the number of lags, $G$ the number of endogenous variables and $q$ the number of exogenous variables.

The generality of (1) comes from at least two features. First, the coetcients are allowed to vary both across units and across time. Second, there are interdependencies among units, since $\mathrm{b}_{i t, l}^{j} \in 0$ for $\mathrm{j} \in \mathrm{i}$ and for any I. Both features constitute the main di Berence with the literature (Holtz-Eakin at al. (1988), Rebucci (1998)) that considers panel VAR models. It is easy to verify that if we set $\mathrm{d}_{i t} \mathrm{v}_{t}=\mathrm{a}_{t} ; \quad \mathrm{b}_{i t}=\mathrm{b}_{t} 8 \mathrm{i} ; \mathrm{u}_{i t}=\tilde{\mathrm{A}}_{t} \mathrm{f}_{i}+»_{i t} \mathrm{~b}_{i t, l}^{j}=0 ; \quad \mathrm{j} \quad \mathrm{i} ; \quad 8 \mathrm{l}$; our speci cation collapses to the one used by Holtz-Eakin et al. (1988).

We rewrite (1) in a stacked regression manner

$$
\mathrm{Y}_{t}=\mathrm{W}_{t}^{\circ}{ }_{t}+\mathrm{U}_{t}
$$

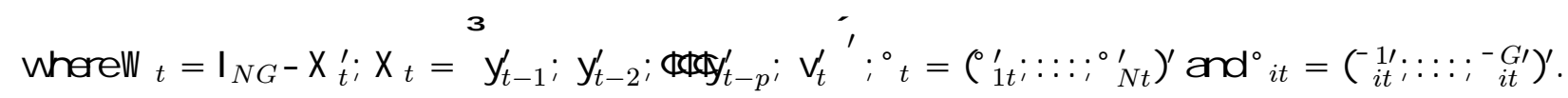
Here $\mathrm{y}_{s}(\mathrm{~s}<\mathrm{t})$ is a NG\{dimensional vector, $\underset{i t}{-g}$ are $\mathrm{k}$ \{dimensional vectors, with $\mathrm{k}=\mathrm{NGp}+\mathrm{q}$, containing, stacked, the $g$ rows of the coe \pm cient matrices $b_{t}$ and $d_{i t}$, while $Y_{t}$ and $U_{t}$ are NG $f 1$ matrices containing the endogenous variables and the random disturbances of the model.

If the ${ }^{\circ}$ it are di Berent for each cross $\{$ sectional unit in di Berent time periods, there is no way to obtain meaningful estimates of them. One possibility is to view each coe \pm cient vector as random with a given probability distribution. We make the following assumptions:

1. For each $\mathrm{i}$, the Gkf 1 vector ${ }^{\circ}$ it has a time invariant and a time varying component, that is

$$
{ }^{\circ}{ }_{i t}=\bigotimes_{l}+{ }_{i t}
$$


2. For each $i$, the Gk $£ 1$ vector of time invariant components $\AA_{\ell}$ follows a normal distribution

$$
\mathbb{R} \gg \mathrm{N}\left(\mathrm{R}_{i} \circledast^{\circledR} \Phi_{i}\right)
$$

where $\mathrm{R}_{i}=\mathrm{I}_{G}-\mathrm{E}_{i}, \Phi_{i}=\mathrm{V}-\mathrm{E}_{i^{-}}{ }_{1} \mathrm{E}_{i}$, and the $\mathrm{G} \notin \mathrm{G}$ matrix $\mathrm{V}$ and the $\mathrm{f} \mathrm{k}$ matrix - ${ }_{1}$ are symmetric and positive de nite. Here $\mathrm{E}_{i}$ is a $\mathrm{k} f \mathrm{k}$ matrix that commutes the $\mathrm{k}$ coe \pm cients of unit $i$ for each of the $G$ equations with those of unit one. Wealso assume that $\operatorname{cov}\left(\AA_{i} ; \AA_{j}\right)=0$ for $\mathrm{i} G \mathrm{j}$.

3. The mean vector ${ }^{\circledR}$ is common to all units and is assumed to have a normal distribution

$$
\text { (B)》N }(1 ; \underline{a})
$$

4. For each i we write the vector of the time varying components as ${ }_{s}{ }_{i t}=\mathrm{R}_{i}{ }_{t}$, where ${ }_{t} t$ is independent of $\AA$ for any $i$. The Gk $f 1$ vector, $t$ evolves according to

$$
{ }_{s}=\mathrm{B}_{, t-1}+\mathrm{e}_{t}
$$

where $\mathrm{B}=1 / 2 \mathrm{\alpha} \mathrm{I}_{G k}$ and, conditional on $\mathrm{U}_{t}$ and $\mathrm{W}_{t}, \mathrm{e}_{t} \gg \mathrm{N}\left(0 ; \S_{\varepsilon}\right)$, with $\S_{\varepsilon}=\mathrm{V}--_{32}$, and -2 is a positive de ${ }^{-}$nite, symmetric matrix. The initial condition is such that $, 0 \gg \mathrm{N}, \widetilde{\sim} ;-0$.

5. Conditional on $\mathrm{W}_{t}$, the vector of random disturbances $\mathrm{U}_{t}$ has a normal distribution

$$
\mathrm{U}_{t} \gg \mathrm{N}\left(0 ; \S_{u}\right):
$$

We assume that $\S_{u}=\S-\mathrm{H}$; where $\S$ is a $\mathrm{N} £ \mathrm{~N}$ matrix and $\mathrm{H}$ is a $\mathrm{G} £ \mathrm{G}$ matrix, both positive de nite and symmetric.

Given the previous assumptions, the structure of the mode (1) can be summarized with the following a \{priori hierarchical scheme

$$
\begin{aligned}
& \left.\mathrm{Y}_{t} \text { j } \quad \mathrm{F}_{t} ; \mathbb{R}_{,}, t\right) \mathrm{N}\left(\mathrm{W}_{t}{ }^{\circledR}+\mathrm{Z}_{t}, t ; \S_{u}\right) \\
& \text { (®) j } \mathrm{F}_{t} \gg \mathrm{N}\left(\mathrm{S}_{N} \circledast, \phi\right) \\
& \text { (B) j } \mathrm{F}_{t} \gg \mathrm{N}\left({ }_{3}^{1} ; \underline{a}\right) \\
& \text {, } t \quad \mathrm{j} \mathrm{F}_{t} \gg \mathrm{N}, \hat{s t \mid t-1} ; \hat{-}_{t \mid t-1}
\end{aligned}
$$

where $\mathrm{F}_{t}$ is the information set at $\mathrm{t}$ (which includes $\mathrm{Y}_{0}$, the presample information, and $\mathrm{W}_{t}$ ); $\mathrm{S}_{N}=$ $\mathrm{e}_{N}-\mathrm{R}_{i} ; \mathrm{Z}_{t}=\mathrm{W}_{t} \mathrm{~S}_{N} ; \Phi=\operatorname{diag}\left(\Phi_{1} ;:: ; \Phi_{n}\right), \hat{\jmath} t \mid t-1=\mathrm{B}, \hat{t-1 \mid t-1} ; \hat{-}_{t \mid t-1}=\mathrm{B} \hat{-}_{t-1 \mid t-1} \mathrm{~B}^{\prime}+\S_{\varepsilon}, \mathrm{e}_{N}$ is a vector of ones of dimension $\mathrm{N}$ and the notation tjt $\mathrm{i} 1$ indicates values at $\mathrm{t}$ predicted with information at $\mathrm{t}_{\mathrm{i}} 1$.

Assumptions 1-4 decompose the parameters vector in 2 components: one is unit speci ${ }^{-} \mathrm{c}$ and constant over time; the other is common across units but varies with time The prior possibility for time\{variation increases the ${ }^{\circ}$ exibility of the speci ${ }^{-}$cation and provides a general mechanism to account for structural shifts without explicitly modelling the source of the shift. The fact that the 
time\{varying parameter vector is common across units does not prevent unit $\left\{\right.$ speci $^{-} \mathrm{c}$ structural shifts, since ${ }^{\circ}$ it can be rewritten as

$$
{ }^{\circ}{ }_{i t}=\left(1 ; 1 / 1 / 1 / 2{ }_{i t-1}+\mathrm{e}_{i t}\right.
$$

where unit speci ${ }^{-} \mathrm{c}$ variations of time occur through the common coe \pm cient $1 / 2$

Assumptions 2 and 3 can be used to recover the vector $\AA$ or the mean coetcient vector $\AA$. In this sense, we can distinguish between " Lindley and Smith (1972). By ${ }^{-}$xed erects we mean the estimation of the vector ${ }^{\circ}{ }_{i t}$, while the term random e eects refers to the estimation of ${ }_{t}{ }_{t}={ }^{\circledR}+{ }_{t}$. For example, in the context of a VAR without interdependencies, (i.e $b_{i t, l}^{j}=0 ;$ j $G$ i), we may be more interested in the relationships among the variables of the system for a "typical" unit, in which case interest centers in the estimation of the random e eect ${ }^{\Phi} t$. If, instead, we are interested in the relationships across units, for example, wishing to ${ }^{-}$nd the erect of a shock in the $g$ variable of unit $j$ on the variables of unit $i$; we better estimate ${ }_{i t}$ for each unit i. In the context of forecasting, we maybeconcerned with point prediction using the average coe \pm cient vector ${ }^{\Phi}$ or in predicting future values of the variables of interest using information available for each unit.

The assumed Kroneker structure for the variance covariance matrices is convenient to nest interesting hypothesis. For instance, when $-_{1}=0$, there is no heterogeneity in the cross sectional dimension of the panel. If $\mathrm{B}=\mathrm{I}_{G k}$, coe \pm cients evolve over time as a random walk, while when $\mathrm{B}=\mathrm{I}_{G k}$ and $-{ }_{2}=0$, the model reduces to a standard dynamic pane model with no time\{variation in the coe \pm cient vector. Finally, when $\mathrm{V}=0$ neither heterogeneity nor time variation are present in the model.

The prior speci cation is fully symmetric in the sense that it is the same regardless of the variables and of the units we are considering. In some applications where it is interesting to consider some prior asymmetries, this restriction may not be needed. In that case we set $\mathrm{E}_{i}=\mathrm{I}_{N}$ so that $\mathrm{R}_{i}=\mathrm{I}_{G}-\mathrm{I}_{N}$ and (3) becomes ${ }_{i t}=\AA_{\imath}+{ }_{t}$ where $\mathbb{Q}_{l} \gg \mathrm{N}\left(\mathbb{R}_{,} \phi\right)$ and the prior distributions for $\circledast$ and, $t$ are the same as before.

As compared to standard BVAR modes, we allow for some degree of a-priori pooling of cross sectional information via the exchangeable prior on ${ }^{\circledR}$. This may be important if there are some similarities in the time series characteristics of the vector of variables considered across units since coe \pm cients of other units may contain useful information for estimating the coe \pm cients of the unit under consideration. A single country VAR with ${ }^{-}$xed coetcients is nested in our speci ${ }^{-}$cation and can be obtained by setting $\mathrm{b}_{i t, l}^{j}=0 ; 8 \mathrm{j} \in \mathrm{i} ; 8 \mathrm{l}$ and letting $\mathrm{x} ; \underline{\mathrm{a}} ; \S_{\varepsilon}$ go to zero.

\section{Posterior Estimates}

\subsection{Fixed effects model}

Given prior information on ${ }^{\circ}$, and assuming that $\widetilde{s}_{0},{ }^{1}$ and the covariance matrices are known, we can obtain the posterior distribution of the parameter vector by combining the likelihood function conditional on $\mathrm{F}_{t}$ with the prior distribution for ${ }_{t}^{\circ}$ in the usual way. From (8) the likelihood is

$$
\mathrm{L}\left(\mathrm{Y}_{t} \mathrm{j}^{\circ} ; \mathrm{F}_{t}\right)=\mathrm{N}\left(\mathrm{W}_{t}{ }^{\circledR}+\mathrm{Z}_{t}, t ; \S_{u}\right)
$$


and the prior, given information at to $t$, is

$$
\mathrm{p}\left({ }^{\circ}{ }_{t} \mathrm{jF}_{t}\right)=\mathrm{N}^{3}{ }^{3}{ }_{t-1} ; \hat{\mathrm{H}}_{t-1}
$$

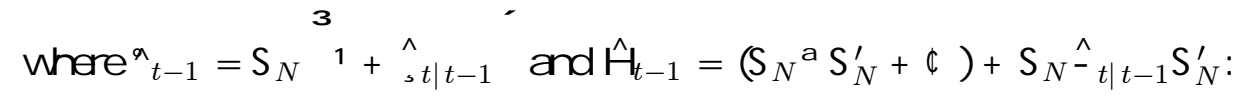

Standard calculations give us that the posterior $\left.1 / 4_{t}^{\circ} \mathrm{j}_{t} ; \mathrm{Y}_{t}\right)$ is normal with mean ${ }_{t}^{\circ *}$ and variance $\mathrm{H}_{t}^{*}$ where:

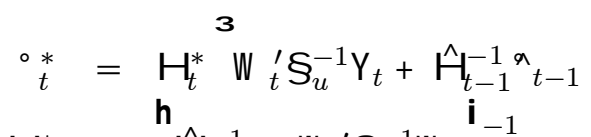

$$
\begin{aligned}
& \mathbf{H}_{t}^{*}=\hat{H}_{t-1}^{-1}+\mathbf{W}_{t}^{\prime} \S_{u}^{-1} \mathbf{W}_{t}{ }^{-1}
\end{aligned}
$$

Hence ${ }_{t}^{\circ *}$ is a standard weighted average of prior and sample information. With a known $\S_{u}$ and starting from initial conditions ${ }^{a}{ }_{0}$ and $\hat{\mathrm{H}_{0}}$ we can also obtain posterior moments for ${ }^{\circ}{ }_{t}$ using the following recursive formulas:

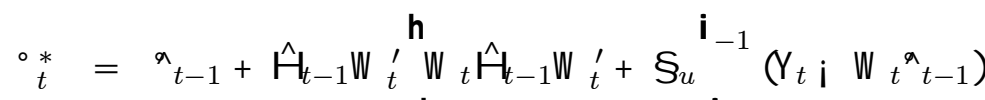

$$
\begin{aligned}
& \mathbf{H}_{t}^{*}=\hat{\mathbf{H}_{t-1}} \mathbf{i} \hat{\mathrm{H}_{t-1}} \mathbf{W}_{t}^{\prime} \mathbf{W}_{t} \hat{\mathrm{H}_{t-1}} \mathbf{W}_{t}^{\prime}+\S_{u}{ }^{i}{ }^{i} \mathbf{W}_{t} \hat{\mathrm{H}_{t-1}}
\end{aligned}
$$

Here information about ${ }_{t}^{\circ *}$ and $\mathrm{H}_{t}^{*}$ is updated in a Kalman ${ }^{-}$Iter fashion.

In some cases attention may be centered in obtaining posterior distributions of ${ }^{\circledR}$ and,$t$ separately. It is straightforward to show that:

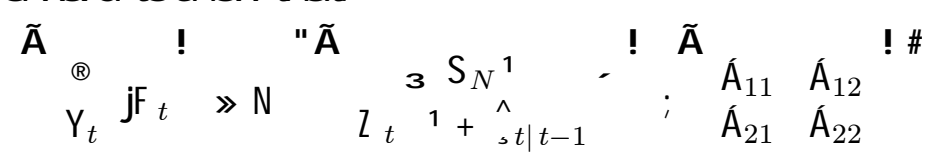

where $\dot{A}_{11}=\left(S_{N}\right.$ a $\left.S_{N}^{\prime}+\Phi\right) ; \dot{A}_{12}=\dot{A}_{11} W_{t}^{\prime} ; \dot{A}_{21}=W_{t} \dot{A}_{11} ; \dot{A}_{22}=W_{t} \dot{A}_{11} W_{t}^{\prime}+Z_{t^{-}}{ }_{t \mid t-1} Z_{t}^{\prime}+\S_{u}$.

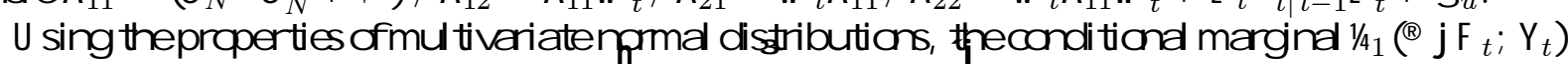
is normal with mean ${ }^{\circledR}=\mathrm{S}_{N}{ }^{1}+\hat{A}_{12} \dot{A}_{22}^{-1} Y_{t} ; Z_{t}{ }^{1}+\hat{s}_{t \mid t-1}$ and variance $V_{\alpha}^{*}=\dot{A}_{11} \mathrm{i} \dot{A}_{12} \dot{A}_{22}^{-1} \dot{A}_{21}$.

Repeating the same argument we obtain that the $e_{3}$ conditional marginal $1 / 4\left(, t, j Y_{t} ; F_{t}\right)$ is normal with mean, ${ }_{t}^{*}=\hat{s}_{t \mid t-1}+\hat{-}_{t \mid t-1} Z_{t}^{\prime} \dot{A}_{22}^{-1} Y_{t} \mathbf{i} Z_{t}{ }^{1}+\hat{s}_{t \mid t-1}$ and variance $-{ }_{t}^{*}=\hat{-t}_{t \mid t-1} \mathrm{i}$

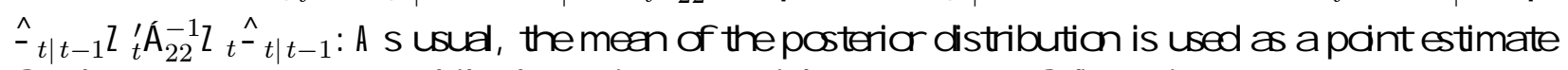
for the parameter vector while the variance provides a measure of dispersion.

For the formulas to be operational we need at timet $=1$ a speci ${ }^{-}$cation for $\S_{u}$ and for the prior distributions of ${ }^{\circledR}$ and,$t$, which in turn requires the speci ${ }^{-}$cation of the matrices $B, \S_{\varepsilon}, \Phi, \underline{a},-0$ and of the vectors ${ }^{1}$ and $\sim$. We will return on this issue in the next section.

\subsection{Random effects model}

When interest centers on the estimation of the mean vector ${ }^{Q}={ }^{2}, t$, we rewrite the model as

$$
\mathrm{Y}_{t}=\mathrm{Z}_{t}{ }^{{ }}{ }_{t}+{ }^{\prime}{ }_{t}
$$


where ${ }_{t}{ }_{t}=\circledast+,{ }_{t}$ and ${ }_{t}=\mathrm{u}_{t}+\mathrm{W}_{t} \mathrm{v}$.

The posterior distributions of $\circledast$ and,$t$ can be obtained by combining the priors and the re spective likelihoods. The sum of the posterior means of $\circledast$ and,$t$ then gives us a point estimate of the mean coetcient vector at each $t$.

Standard manipulations give us that the posterior $1 / 4\left(\circledast j \mathrm{Y}_{t} ; \mathrm{F}_{t}\right) \gg \mathrm{N}\left(\circledast^{*} ; \underline{\mathrm{a}}^{*}\right)$ and the posterior $1 / 4\left(, t, j Y_{t} ; F_{t}\right) \gg N\left(,{ }_{t}^{*} ;-{ }_{t}^{*}\right)$ where

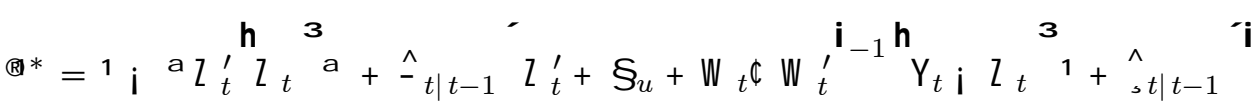

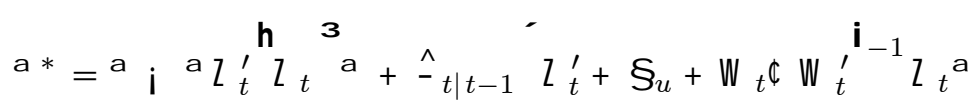

while the expressions for ${ }_{t}^{*}$ and $-{ }_{t}^{*}$ are the same as before This implies that the posterior ${ }_{1 / 4}\left({ }_{t}{ }_{t} \mathrm{j}\right.$ $\left.\mathrm{Y}_{t} ; \mathrm{F}_{t}\right) \gg \mathrm{N}\left({ }_{t}^{ \pm *} ; \mathrm{H}_{t}^{*}\right)$ where

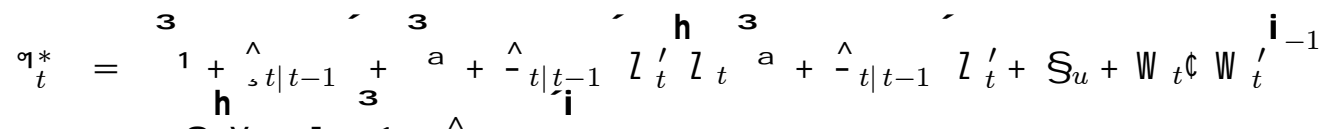

$$
\begin{aligned}
& \text { f } \mathrm{Y}_{t} \mathrm{i} \mathrm{Z}_{t}{ }^{1}+\hat{\jmath_{t} \mid t-1}
\end{aligned}
$$

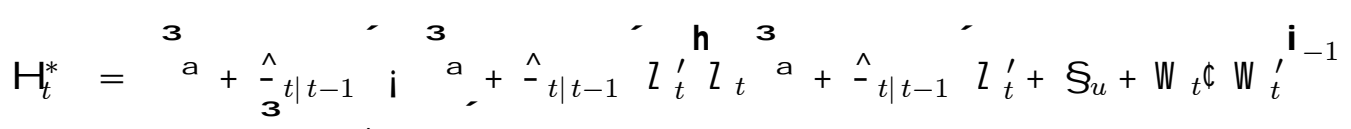

$$
\begin{aligned}
& f Z_{t} \underline{a}+\hat{-}_{t \mid t-1}
\end{aligned}
$$

\section{Setting up the priors}

For the formulas described in the previous section to be operational, we need to specify the vector $3=\left(1 ; \sim_{o} ;-{ }_{o} ; \S_{u} ; \S_{\varepsilon} ; \mathrm{B} ; \underline{\underline{a}} ; \Phi\right)$. The results of section 3 were obtained under the assumption that this vector of parameters was known. In practice, this is hardly the case: to get posterior distributions for the parameters we need to make assumptions on the ${ }^{3}$ vector and to obtain marginal posteriors we need to integrate nuisance parameters out of the joint posterior density. This integration, in general, is di \pm cult, even with brute force numerical methods, given the large number of parameters typically contained in ${ }^{3}$.

There are several ways to proceed. One is to assume a di @use prior on some of the components of the parameter vector, while still assuming that others are known. Another is to specify a Litterman-type prior wherethe unknown elements of ${ }^{3}$ depend on a small vector of hyperparameters to be estimated from the data in Empirical Bayes fashion. The third is to assume explicit prior distributions for the parameter vector and proceed directly to the numerical integration using Markov Chains-Monte Carlo methods. We examine these approaches in turn.

\subsection{Diffuse Priors}

Imposing di @use priors is interesting in our context as a way to describethe ignorance of a researcher on some aspects of the prior distribution. It is well known (see Zellner (1971)) that a joint di ßuse 
prior for all the elements of ${ }^{3}$ leads to posteriors which contain the sample information summarized in a least square fashion. Also, as shown by Kadiyala and Karlsson (1997), such prior produces posterior dependence among the coetcients of di Berent equations, i.e. the joint posterior for the NGK $£ 1$ vector of coe \pm cients does not factor into the product of the posterior for the $k$ coe \pm cients of each of the NG equations. Here we concentrate attention on two special cases of interest; one where there is no information on the location of the mean of the unit speci ${ }^{-}$c eBect $^{i}{ }^{-1}=0^{4}$ and one where there is no information on the time varying component of the coe + cients either at time zero $-{ }_{0}^{-1}=0$ or at a particular point in time $\hat{-}_{t \mid t-1}^{-1}=0$. All other components of the vector of parameters are assumed to be known.

\subsubsection{Case 1: I gnorance about $\circledast$}

When the prior distribution of the second stage of the hierarchy is proportional to a constant, the posterior distribution changes according to the following proposition:

Proposition 4.1 Given the prior $(8)$, if $\underline{a}^{-1}=0$; conditional on $Y_{t}$ and $F_{t}$,

(i) The posterior distribution $1 / 4\left(\circledast \mathrm{j} \mathrm{Y}_{t} ; \mathrm{F}_{t}\right)$ is normal with mean $\mathbb{R}^{*}$ and variance a $* *$ where

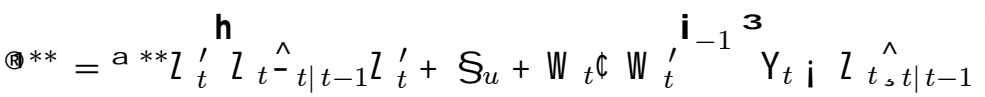

$$
\begin{aligned}
& \underline{\mathrm{a} * *-1}=\mathrm{Z}_{t}^{\prime} \mathrm{Z}_{t^{-}{ }^{-}{ }_{t \mid t-1}} \mathrm{Z}_{t}^{\prime}+\S_{u}+\mathrm{W}_{t} \pitchfork \mathrm{W}_{t}^{\prime}{ }^{\mathrm{i}} \mathrm{Z}_{t}
\end{aligned}
$$

(ii) The posterior distribution $1 / 4\left({ }^{\circledR} j \mathrm{Y}_{t} ; \mathrm{F}_{t}\right)$ is normal with mean ${ }^{\circledR *}$ and variance $\mathrm{V}_{\alpha}^{* *}$ where

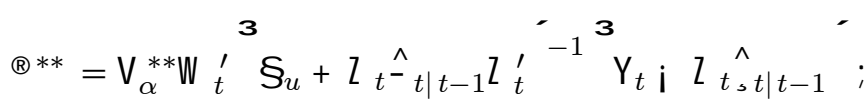

$$
\begin{aligned}
& \mathrm{V}_{\alpha}^{* *-1}=\mathrm{W}_{t}^{\prime} \S_{u}+\mathrm{Z}_{t^{-}} \hat{-}_{t \mid t-1} \mathrm{Z}_{t}^{\prime}{ }^{-1} \mathrm{~W}_{t}+\mathrm{F}
\end{aligned}
$$

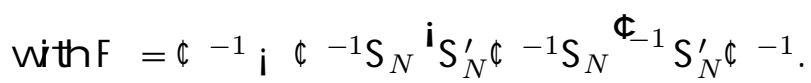

(iii) The posterior distribution of,$t$ is equal to the prior, i.e,

$$
1 / 2\left(, t, j Y_{t} ; X_{t}\right)=p\left(, t ; X_{t}\right):
$$

(The proof of all propositions is in the appendix).

Notice that the di $\circledast_{\text {use prior on }} \circledast$ does not allow to update the prior information we have on ${ }_{t}$. In fact, in this case, the posterior distribution of ${ }_{t}{ }_{t}$ does not depend on the prior for ${ }_{t}$. To see this note that, with $\underline{a}-1=0$; we have that $\hat{\mathrm{H}_{t-1}} \mathrm{~S}_{N} \underline{\underline{a}}+\hat{\bar{t}_{t \mid t-1}} \mathrm{~S}_{N}^{\prime}+\phi=\mathrm{F}^{-1}$ and using

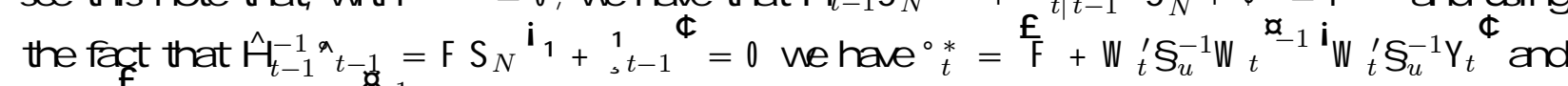
$\mathrm{H}_{t}^{*}={ }^{\ddagger} \mathrm{F}+\mathrm{W}_{t}^{\prime} \S_{u}^{-1} \mathrm{~W}_{t}{ }^{-1}$ where no prior information on,$t$ is involved. 
4.1.2 Case 2: Ignorance about , $t$

There are two simple ways of attaching a di \&use prior to the time varying component of the coe \pm cient vector. One possibility is to consider lack of information at time zero $\left(-\frac{-1}{0}=0\right)$. When the prior distribution for, 0 is proportional to a constant, given the autoregressive structure for,$t$, and provided $1 / 2<1$, the process tends to "forget" the initial condition. In other words, subsequent realizations of , $t$ make less and less uncertain our information on the time varying component of the coe \pm cients so that $-{ }_{0}^{-1}=0$ does not imply $\hat{-}_{t \mid t-1}^{-1}=0$ at all points in time and, for large enough $\mathrm{T}$, the posterior for ${ }^{\circledR}$ and,$t$ is the one presented in section 3 .

Another possibility is to set $\S_{\varepsilon}^{-1}=0$. To implement this di @use prior, we assume $-{ }_{2}^{-1}=0$. Notice that $\hat{-}_{t \mid t-1}=\mathrm{B} \hat{-}_{t-1 \mid t-1} \mathrm{~B}^{\prime}+\S_{\varepsilon}$. Therefore if $\S_{\varepsilon}^{-1}=0, \hat{-}_{t \mid t-1}^{-1}=0$. In this case it is possible to prove the following result

Proposition 4.2 Given the prior (8), if $\S_{\varepsilon}^{-1}=0$; then

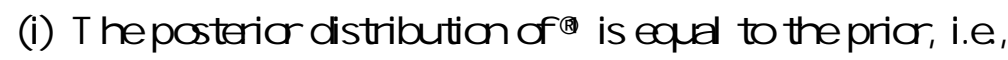

$$
1 / 4\left(\circledast \mathrm{j} \mathrm{Y}_{t} ; \mathrm{X}_{t}\right)=\mathrm{p}\left(\circledast \mathrm{j} \mathrm{X}_{t}\right)
$$

(ii) The posterior distribution $1 / 4\left(\circledR j \mathrm{j} \mathrm{Y}_{t} ; \mathrm{F}_{t}\right)$ is normal with mean ${ }^{\circledR *}$ and variance $\mathrm{V}_{\alpha}^{* *}$ where

$$
\begin{aligned}
& \left.{ }^{\circledR}\right)^{*}=\mathrm{V}_{\alpha}^{* *} \mathrm{~W}_{t}^{\prime} \mathrm{S}^{3} \mathrm{Y}_{t} \mathrm{i} \mathrm{Z}_{t, \hat{t} \mid t-1}+{ }^{\mathrm{i}} \mathrm{S}_{N} \underline{\mathrm{a}} \mathrm{S}_{N}^{\prime}+\phi^{\phi}{ }_{-1} \mathrm{~S}_{N}{ }^{\mathrm{i}} \text {; } \\
& \mathrm{V}_{\alpha}^{* *-1}=\mathrm{W}_{t}^{\prime} \mathrm{TW}_{t}+{ }^{\mathrm{i}} \mathrm{S}_{N} \underline{\mathrm{a}} \mathrm{S}_{N}^{\prime}+\phi^{\phi_{-1}} \\
& \text { and } \mathbf{T}=\S_{u}^{-1} \mathbf{i} \S_{u}^{-1} \mathbf{Z}_{t}{ }^{\mathrm{i}} \mathbf{Z}_{t}^{\prime} \S_{u}^{-1} \mathbf{Z}_{t}^{\boldsymbol{\phi}_{-1}} \mathbf{Z}_{t}^{\prime} \S_{u}^{-1}
\end{aligned}
$$

(iii) The posterior distribution $\left.1 / 4(, t) \mathrm{Y}_{t} ; \mathrm{F}_{t}\right)$ is normal with mean ${ }_{t}^{* *}$ and variance - ${ }_{t}^{* *}$ where

$$
\begin{aligned}
& { }_{, t}^{* *}=-{ }_{t}^{* *}{ }^{\mathrm{n}} \mathrm{Z}_{t}^{\prime}{ }^{\mathrm{f}} \mathrm{W}_{t}{ }^{\mathrm{i}} \mathrm{S}_{N}{ }^{\underline{a}} \mathrm{~S}_{N}^{\prime}+\phi^{\Phi} \mathrm{W}_{t}^{\prime}+\S_{u}{ }^{\mathrm{a}_{-1}}\left(\mathrm{Y}_{t} \mathbf{i} \mathrm{Z}_{t}{ }^{1}\right)^{\mathrm{o}} \\
& -{ }_{t}^{* *-1}=Z_{t}^{\prime}{ }^{\ddagger} W_{t}{ }^{\mathrm{i}} S_{N} \text { a } S_{N}^{\prime}+\phi^{\Phi} W_{t}^{\prime}+\S_{u}{ }^{\alpha_{-1}} Z_{t}
\end{aligned}
$$

The assumption $\S_{e}^{-1}=0$ implies that $\hat{-}-1$ t|t-1 $=0$, at all points in time. This implication is unreasonable or, at least, excessively myopic, because it prevents researchers to learn from past realizations of,$t$ and to be less uncertain on its mean as times goes by. The assumption $\hat{-}-1=0$ can be more realistic if we attach this in ${ }^{-}$nite uncertainty to the coe \pm cients only at a particular point in time (let's say, $\mathrm{t}=\mathrm{t}_{o}$ ), perhaps to take care of a structural break, after which the process restarts and behaves as it did before the break.

It is worth noting that in both cases 1 and 2 , the posterior mean and variance for ${ }_{t}{ }_{t}$ are the same as those obtained when only prior information on $®$ is used. This is not surprising if we write (8) as a three stage hierarchy

$$
\begin{aligned}
& \mathrm{Y}_{t} \quad \mathrm{j} \quad \mathrm{F}_{t} ;{ }_{t} \gg \mathrm{N}\left(\mathrm{W}_{t}^{\circ}{ }_{t} ; \S_{u}\right)
\end{aligned}
$$

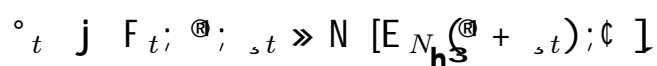

$$
\begin{aligned}
& (\circledast+, t) \quad \mathrm{j} \mathrm{F}_{t} ;{ }^{1} ; \hat{\jmath}, t\left|t-1 》 \mathrm{~N}^{1}+\hat{\jmath} t\right| t-1 ; \underline{a}+\hat{-}_{t \mid t-1} \mathrm{i} \text { : }
\end{aligned}
$$


Assuming ${ }^{a-1}=0$ or $\S_{\varepsilon}^{-1}=0$ is equivalent to assume a di ®use prior on the third stage of the hierarchy.

\subsection{Litterman-type prior}

Next, we modify the so-called Minnesota prior to account for the presence of multiple units in the VAR. The Minnesota prior, described in Litterman (1986), Doan, Litterman and Sims (1984), Ingram and Whiteman (1995), Ballabriga, et al. (1998) among others is a way to account for the near nonstationarity of many macroeconomic time series and, at the same time, to weakly reduce the dimensionality of a VAR model. Given that the intertemporal dependence of the variables is believed to be strong, the prior mean of the VAR coe \pm cients on the ${ }^{-}$rst own lag is set equal to one and the mean of remaining coe \pm cients is equal to zero. The covariance matrix of the coe \pm cients is diagonal (so we have prior | and posterior | independence between equations) and the elements are speci ${ }^{-}$ed in a way that coe \pm cients of higher order lags are likely to be close to zero (the prior variance decreases when the lag length increases). Moreover, since most of the variations in the VAR variables is accounted for by own lags, coe \pm cients of variables other than the dependent one are assigned a smaller relative variance. The prior on the constant term, other deterministic and exogenous variables is di ßuse. Finally, the variance-covariance matrix of the error term is assumed to be ${ }^{-}$xed and known.

For a panel VAR setup we introduce the following modi ${ }^{-}$cations. The covariance matrices - ${ }_{o} ; \underline{a} ; \Phi$, areassumed to havethesamea-priori structure Take, for example, $₫=\operatorname{diag}\left(\phi_{1} ;:: ; ; \Phi_{n}\right)$, where $\phi_{i}=\mathrm{V}-\mathrm{E}_{i^{-}}{ }_{1} \mathrm{E}_{i}$.

The matrix $-{ }_{1}$ is assumed to be diagonal and its elements have the following structure:

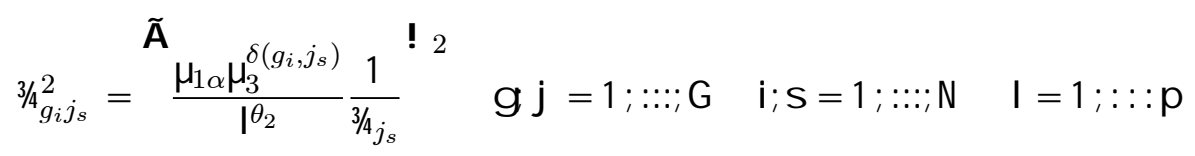

where $\pm\left(g_{i} ; j_{s}\right)=0$ if $\mathrm{i}=\mathrm{s}$ and 1 otherwise and

$$
3 / \frac{2}{g} m=\left(\mu_{1} \alpha \mu_{1}\right)^{2} \quad \mathrm{~m}=1 ; ;: ; ; q
$$

Here, $g_{i}$ represents equation $g$ of unit $i, j_{s}$ theendogenous variablej of unit $s, I$ thelag, m exogenous or deterministic variables.

The hyperparameter $\mu_{1}$ controls the tightness of beliefs for the vector ${ }^{\circledR}, \mu_{2}$ the rate at which the prior variance decays with the lag; $\mu_{3}$ the degree of uncertainty for the coe \pm cients of the variables of unit $s$ in the equations of unit $i ; \mu_{4}$ the degree of uncertainty of the coe \pm cients of the exogenous variables and $3 / y_{s}$ are the diagonal elements of the matrix $\S_{u}$ used as scale factors to account for di eerences in units of measurement. Also, assume that $\mathrm{V}=\mathrm{H}$ (see equation (7)). Notice that we don't have prior independence between equations. Hence our prior information speci $^{-}$es that, for example, the coetcient on lag 1 of the GNP equation for the US may have some relationship with the same coe \pm cient in the PRICE equation for US. Moreover, we have not speci ${ }^{-}$ed a hyperparameter which controls the overall tightness of beliefs because the randomness of the coe \pm cients depends on $\mathbb{Q}$ and,$t$ and we parametrize the uncertainty in each of them separately. 
Finally, there is no distinction between own versus other countries variables. Because of this $V$ and - 1 are common to all units and the prior has a symmetric structure (see Sims and Zha (1998)).

The structures for $\underline{\underline{a}}$ and $-{ }_{o}$ are similar with $\mu_{1 \alpha}$ being replaced by $\mu_{\bar{\alpha}}$ and $\mu_{1 \lambda}$, respectively.

To complete the speci ${ }^{-}$cation we need to have a measure the elements of the matrix $\mathrm{H}$ and of the $3 / 45$. Following Litterman, these parameters are estimated from the data to tune up the prior to the speci ${ }^{-} \mathrm{c}$ application.

The prior time \{varying features of the model are determined by specifying the matrices $B, \S_{\varepsilon}$. Weassumethat B is diagonal and that each of the $\mathrm{f} \mathrm{k}$ diagonal blocks $\mathrm{B}_{g}$ satis ${ }^{-} \mathrm{es}: \mathrm{B}_{g}=\operatorname{diag}\left(\mu_{5}\right)$. Furthermore, we assume $\S_{\varepsilon}=\mu_{6}{ }^{-}{ }_{o}$. Here $\mu_{5}$ controls the evolution of the law of motion of,$t$ and $\mu_{6}$ the heteroskecasticity in the coe \pm cients. Note that a time invariant model is obtained by setting $\mu_{5}=1$ and $\mu_{6}=0$. Homoschedastic time variations are obtained by setting $\mu_{6}=0$.

Finally, we assume that the $\mathrm{kf} 1$ vectors ${ }^{1} g$ and $\sim$ og have the following structures:

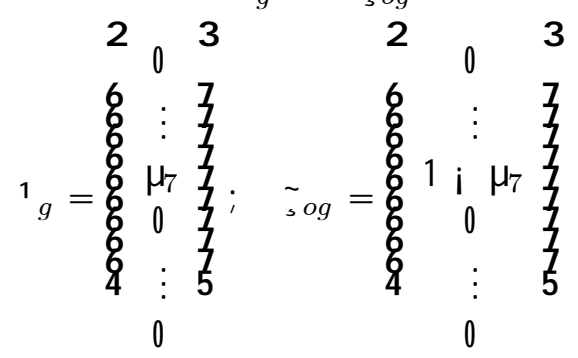

where ${ }^{1} g$ and $\sim_{s}$ are the gth \{elements of the mean vectors ${ }^{1}$ and $\tau_{o}$ and $\mu_{7}$ controls the prior mean on the ${ }^{-}$rst own lag coe \pm cient of the dependent variable in equation $g$ for unit $i$.

Summing up, our prior information is a function of a 9 \{dimensional vector of hyperparameters $£=\left(\mu_{1} ; \mu_{1} ; \mu_{1} ; \mu_{2} ; \mu_{3} ; \mu_{4} ; \mu_{5} ; \mu_{6} ; \mu_{7}\right)$. Estimates of $£$ can be obtained by maximizing the predictive density of the model as in Doan, Litterman and Sims (1984). Posterior distributions for the parameters are then obtained by plugging-in the resulting estimates for ${ }^{1} ;$, ${ }_{0} ;-{ }_{o} ; \S_{u} ; \S_{\varepsilon} ; \mathrm{B} ; \underline{\underline{a}} ; \varnothing$ in the formulas we have derived in section 3 in an empirical Bayes fashion (see eg. Berger (1985)).

Compared with Ballabriga et al. (1998), who used a Minnesota prior on a pane VAR model for the Spanish, German and French economies, our speci ${ }^{-}$cation allows for unit speci ${ }^{-} \mathrm{c}$ time variations in the variance of the process $\left(\mu_{6} \in 0\right)$; it separates the prior information for the time and the individual component (they have one parameter in place of $\mu_{1} ; \mu_{1} ; \mu_{1}$ ) and introduces a further level of uncertainty by specifying a prior for ${ }^{\circledR}$ Furthermore, our prior speci ${ }^{-}$cation is symmetric and it allows for a-priori pooling of the information present in the cross sectional dimension of the panel. None of these features is present in their speci- cation.

\subsection{Informative priors}

When the prior for the vector of parameters is informative, the posterior distribution for the parameter vector does not have an analytical closed form. Nevertheless, we can implement a hierarchical Bayes analysis using a sampling \{based approach, such as the Gibbs sampler, (see eg. Geman and Geman (1984), Gelfand and Smith (1990), Gelfand and al. (1990) among others).

The basic idea of the approach is to construct a (computable) Markov chain on a general state space such that the limiting distribution of the chain is the joint posterior of interest. Suppose we 
have a parameter vector \# with $\mathrm{k}$ components $\left(\#_{1} ; \#_{2} ;:: ; ; \#_{k}\right)$ and that the posterior distributions $1 / 4\left(\#_{j} \mathrm{j} \#_{s} ; \mathrm{s} \in \mathrm{j}\right)$ areavailable. Then theal gorithm works as follows. Westart from arbitrary values for $\#_{1}^{(o)} ; \#_{2}^{(o)} ; \ldots ; ; \#_{k}^{(o)}$. Setting $\mathrm{i}=1$; we scycle through the conditional distribstions sampling $\#_{1}^{(1)}$ from $1 / 4 \#_{1} \mathrm{j} \#_{2}^{(o)} ;: \ldots ; \#_{k}^{(o)} \quad \#_{2}^{(i)}$ from $1 / 4 \#_{2} \mathrm{j} \#_{1}^{(1)} ;: \ldots ; ; \#_{k}^{(o)}$ up to $\#_{k}^{(i)}$ from $1 / 4 \#_{k} \mathrm{j} \#_{1}^{(1)} ;: \ldots: ; \#_{k-1}^{(1)}$ : Next, we set $\mathrm{i}=2$ and repeat,the cycle. After iterating on this cycle, say, $M$ times, the sample value $\#^{(M)}=\#_{1}^{(M)} ; \#_{2}^{(M)} ;: \ldots ; \#_{k}^{(M)}$ can be regarded as a drawing from the true joint posterior density. Once this simulated sample has been obtained, any posterior moment of interest or any marginal density can be estimated, using the ergodic theorem. Convergence to the desired distribution can be checked as suggested in Gelfand and Smith (1990).

In order to apply the Gibbs sampler to our pane VAR model we need to specify prior information so that the conditional posterior distribution for components of the parameter vector can be obtained analytically. Recall that our hierarchical model is given by:

$$
\begin{aligned}
\mathrm{Y}_{t} & =\mathrm{W}_{t} \circledast+\mathrm{Z}_{t, t}+\mathrm{U}_{t} ; \\
\circledR & =\mathrm{S}_{N} \circledast+{ }^{\circledR}{ }_{i} \\
\circledR & =1+\mathrm{v} \\
{ }^{\circledR} & =\mathrm{B}, t-1+\mathrm{e}_{t}
\end{aligned}
$$

where $\mathrm{u}_{t} \gg \mathrm{N}(0 ; \S-\mathrm{H}) ;{ }_{i} \gg \mathrm{N}\left(0 ; \mathrm{V}-\mathrm{E}_{i^{-}}{ }_{1} \mathrm{E}_{i}\right) ; \mathrm{V} \gg \mathrm{N}(0 ; \underline{a}) ;, o \gg \mathrm{N}\left(0 ; \mathrm{V}-{ }_{2}\right) \mathrm{e}_{t} \gg$ $\mathrm{N}\left(0 ; \mathrm{V}-{ }^{\prime}-{ }_{2}\right)$ and ' is the tightness on time variation: if ' $=0$ and $\mathrm{B}=\mathrm{I}$ then, is time invariant. We assume that the covariance matrices are independent, that $\mathrm{V}^{\prime}{ }^{\mathrm{a}},{ }^{\prime}{ }^{\prime}$, and ${ }^{1}$ are known and that $\S \gg \mathrm{iW}_{N}\left(3 / 8 ; \mathrm{M}_{o}\right), \mathrm{H} \gg \mathrm{iW}_{G}\left(\mathrm{~h}_{o} ; \mathrm{P}_{o}\right) ;-{ }_{1} \gg \mathrm{iW}_{k}\left(\mathrm{~W}_{1} ; \mathrm{W}_{1}\right)$, and $-{ }_{2} \gg \mathrm{iW}_{k}\left(\mathrm{~W}_{2} ; \mathrm{W}_{2}\right)$, where the notation (c) » $\mathrm{iW}_{p}(\mathrm{v} ; Z)$ means that the symmetric positive de- nite matrix (c) follows a $\mathrm{p}$ \{dimensional inverted Wishart distribution with $\mathrm{v}$ degrees of freedom and scale matrix Z: We also assume that for each of these distributions the degrees of freedom and the scale matrix are known. These assumptions are inconsequential and the analysis goes through, even when consistent estimates are substituted for the true ones.

Given this prior information, the posterior density of the parameter vector $\#=(\circledast, \S ; H ; \circledast,-1$, $\left.\mathrm{f}, t \mathrm{~g}_{t=0}^{T} ;-{ }_{2}\right)$ is given by

$$
1 / 4\left(\# \mathrm{j} Y_{T} ; F_{T}\right) / f\left(Y_{T} \mathrm{j} \#_{T} ; F_{T}\right) p\left(\# j F_{T}\right)
$$

where $Y_{T}=\left(Y_{1} ;: ; ; Y_{T}\right)$ is the sample data and $p\left(\# j F_{T}\right)$ is the prior information available at $\mathrm{T}$.

Given the di \pm culty to obtain marginal posteriors directly from the integration of (19), we iterate on the conditional distributions of the parameters, which can easily be obtained from the conditional posterior (19). To deal with the presence of time varying parameters we adapt the results of Carter and Khon (1994) and Chib and Greenberg (1996). In fact, conditional on $f,{ }_{t} g_{t=0}^{T}$, the distribution of the remaining parameters can be derived without di \pm culty. Let $\tilde{\mathrm{A}}_{-x}$ be the vector \# containing all the parameters but $x$. Then the conditional distributions for parameters other than $f, t g$ are:

$$
-_{1} \quad \mathrm{j} \quad \tilde{A}_{-} ; \mathrm{Y}_{T} ; \mathrm{F}_{T} \gg \mathrm{iW}_{k}^{3} \mathrm{~W}_{1}+\mathrm{NG} ; \widehat{W}_{1}
$$




$$
\begin{aligned}
& { }_{-2} \quad \mathrm{j} \quad \tilde{A}_{-} ; \mathrm{Y}_{T} ; \mathrm{F}_{T} \gg \mathrm{iW}_{k_{3}} \mathrm{~W}_{2}+\mathrm{TG} ; \widehat{W}_{2} \text {, } \\
& \S \text { j } \tilde{A}_{-\Sigma} ; Y_{T} ; F_{T} \gg i W_{N} 3 / 8+G T ; \hat{M}_{o} \text {, } \\
& \mathrm{H} \quad \mathrm{j} \quad \tilde{\mathrm{A}}_{-H} ; \mathrm{Y}_{T} ; \mathrm{F}_{T} \gg \mathrm{iW}_{3} \mathrm{~h}_{o}+\mathrm{NT} ; \hat{\mathrm{P}_{o}} \\
& \text { ( } \mathrm{j} \quad \tilde{A}_{-\alpha} ; Y_{T} ; F_{T} \gg \mathrm{N}_{3}\left(\mathbb{R}_{\alpha}\right. \text {, } \\
& \text { (B) j } \tilde{\mathrm{A}}_{-\bar{\alpha}} ; \mathrm{Y}_{T} ; \mathrm{F}_{T} \gg \mathrm{N} \quad \mathbb{B}^{*} ; \hat{\mathrm{V}}^{*}
\end{aligned}
$$

where the expressions for $\widehat{W}_{1} ; \widehat{W}_{2} ; \hat{M_{o}} ; \widehat{P_{o}} ; \widehat{\mathbb{V}} ; \hat{\mathrm{V}_{\alpha}} ; \widehat{\mathrm{V}}^{*}$ are given in the appendix.

Following Chib (1996) the parameter vector, $t$ can be included in the Gibbs sampler via the distribution $1 / 4\left(, ; \cdots: ;, T, j \mathrm{Y}_{T} ; \mathrm{F}_{T} ; \tilde{\mathrm{A}}_{T}\right)$ where $\tilde{\mathrm{A}}_{t}{ }^{\prime} \#_{-\left\{\lambda_{t}\right\}_{t}}$. We can re\{write such a distribution as

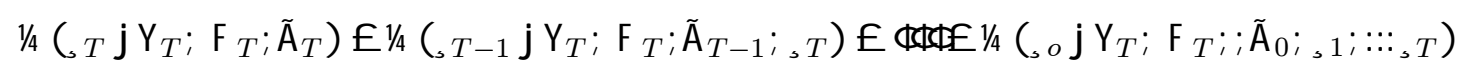

A draw from 3 the joint distribution can be obtained by drawing $\widetilde{\Omega}_{T}$ from $\left.1 / 4,{ }_{T} \mathrm{j}_{T} ; \mathrm{F}_{T} ; \tilde{\mathrm{A}}_{T}\right)$; then $\widetilde{s-1}$ from $1 / 4, T-1, \mathrm{j}_{T} ; \mathrm{F}_{T} ; ; \tilde{\mathrm{A}}_{T-1} ; \tilde{s T}$ and so on. Let, ${ }^{s}=(, s ;:: ;, T)$ and $\mathrm{Y}^{s}=\left(\mathrm{Y}_{s} ;:: ; \mathrm{Y}_{T}\right)$ for $\mathrm{S} \cdot \mathrm{T}$. The density of the typical term in (21) is

$$
\begin{aligned}
& 1 / 4,{ }_{3}, \mathrm{j}_{T} ; \mathrm{F}_{T} ; \tilde{\mathrm{A}}_{t, 2}{ }^{t+1}
\end{aligned}
$$

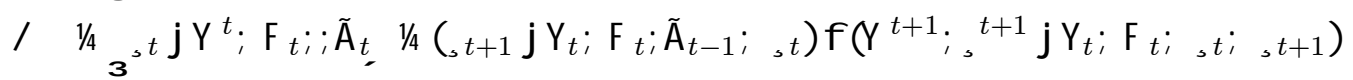

$$
\begin{aligned}
& \text { / } 1 / 4, t \mathrm{j}^{t} ; \mathrm{F}_{t} ; \tilde{\mathrm{A}}_{t} 1 / 4\left(\mathbb{}_{t+1} \mathrm{jF}_{t} ; \tilde{\mathrm{A}}_{t-1} ;{ }_{,}\right)
\end{aligned}
$$

The last row follows from the fact that, conditional on,$t+1$; the joint density of $\left(\mathrm{Y}^{t+1} ;{ }_{,}{ }^{t+1}\right)$ is independent of,$t$ and, conditional on $, t, t_{t+1}$ is independent of $Y_{t}$.

The second density of (22) in Gaussian with moments $1 / 3 t$ and $\S_{\varepsilon_{3}}$ The ${ }^{-}$rst was derived in section 3, and it is Gaussian with mean $\hat{\jmath}_{t \mid t}=\hat{s}_{t \mid t-1}+\hat{-}_{t \mid t-1} Z_{t}^{\prime} \dot{\mathrm{A}}_{22}^{-1} \mathrm{Y}_{t} \mathbf{i} Z_{t}{ }^{1} \mathbf{i} Z_{t}, \hat{t} \mid t-1$ and

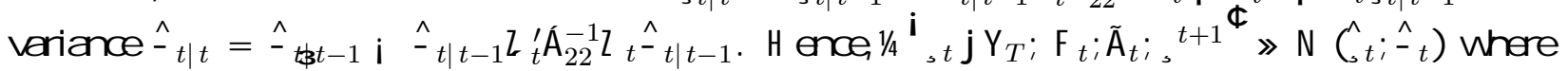

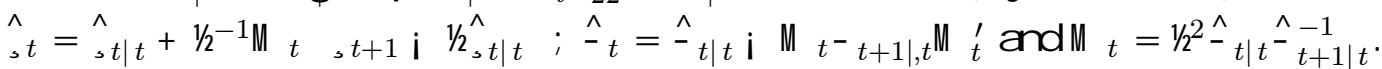

To be concrete the following algorithm can be used to sample $f, t g$ : ${ }^{-} r s t$, starting from given

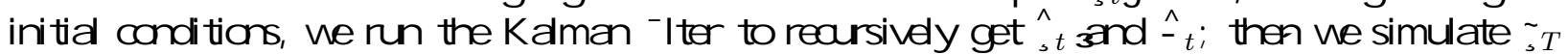

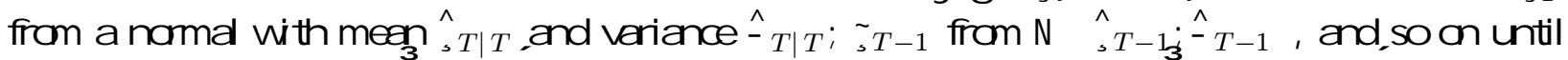

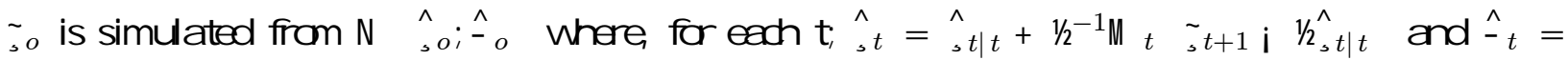
$\hat{-}_{t \mid t}$ i $\mathbf{M}_{t^{-}} \hat{}_{t+1 \mid, t} \mathbf{M}_{t}^{\prime}$.

One special case of the setup described in this subsection deserve some attention. Suppose informative priors on all the parameters except that on $\mathrm{H}$, whose prior is now di \&use, so that the prior for $\S_{u}$ is di @use as well. Then the setup resembles the Normal-Di @ise prior of Kadiyala and Karlsson (1997) and implies that posterior dependence among the coe \pm cients of di Berent equations obtains even when there is prior independence. Hence, the major di Berence of our prior with the speci ${ }^{-}$cation used by these authors is that we use a three stage hierarchy, so that both the mean and the variance of ${ }_{t}^{\circ}$ are random variables, while they take the mean and the variance of ${ }_{t}^{\circ}$ to be 
- xed. Note also that our speci ${ }^{-}$cation does not restrict $\S_{u}$ to be diagonal and therefore permits complicated interactions among variables within and across countries.

Finally, it is worth mentioning that in all the setups we have considered in this section, our prior speci ${ }^{-}$cation maintains a knoneker structure for the statistical model. Such a speci ${ }^{-}$cation is useful since, on one hand, it allows to handle the computations for relatively large systems in a simple fashion and, on the other, imposes symmetry restrictions which appear to be desirable in an unrestricted VAR system of thetype examined here Clearly these restrictions may beinappropriate for structural or restricted VAR systems and alternative speci ${ }^{-}$cations, along the lines of Sims and Zha (1998), should be used.

\section{$5 \quad$ Forecasting}

Once posterior estimates are obtained, forecasts can be computed. In order to obtain multistep forecasting formulas for a pane VAR and to compute turning points probabilities, it is convenient to rewrite (1) in a companion VAR(1) form

$$
\mathrm{Y}_{i t}={ }_{j=1}^{\mathrm{X}} \mathrm{B}_{i t}^{j} \mathrm{Y}_{j t-1}+\mathrm{D}_{i t} \mathrm{Z}_{t}+\mathrm{U}_{i t}
$$

where $Y_{i t}$ and $U_{i t}$ are $G p f 1$ vectors, $B_{i t}^{j}$ is a $G p f$ Gp matrix and $D_{i t}$ is a Gpf q matrix.

Stacking for $\mathrm{i}$, and repeatedly substituting we have

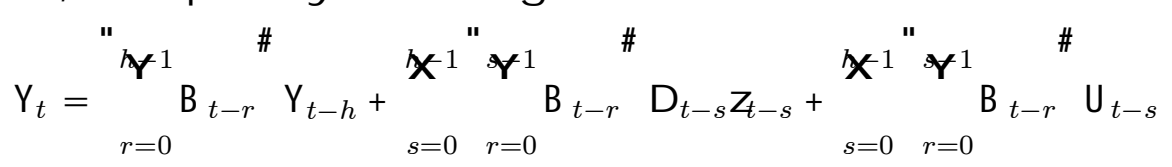

or

$$
\mathrm{y}_{t}=\mathrm{J}{ }_{r=0}^{n-1} \mathrm{~B}_{t-r} \mathrm{Y}_{t-h}+{ }_{s=0}^{\not \mathcal{X}^{-1}} \bigodot_{s t} \mathrm{D}_{t-s} \mathrm{Z}_{t-s}+{ }_{s=0}^{\mathcal{X}^{-1}} \bigodot_{s t} \mathrm{u}_{t-s}
$$

where $\bigcirc_{s t}={ }^{Q_{s-1}} \mathrm{~B}_{t-r}$, and $\mathrm{J}=\mathrm{I}_{N}-\mathrm{J}_{1}, \mathrm{~J}_{1}=\left[\begin{array}{ll}\mathrm{I}_{G} & 0\end{array}\right]$ and $\mathrm{J}$ is a selection matrix such that $\mathrm{J} \mathrm{Y}_{t}=\mathrm{y}_{t}, \mathrm{~J} \mathrm{U}_{t}=\mathrm{U}_{t}$ and $\mathrm{J}$ ' $\mathrm{J} \mathrm{U}_{t}=\mathrm{U}_{t}$. The expression in (25) can be used to compute the $\mathrm{h}$ \{steps ahead forecast of the $\mathrm{NG}$ \{dimensional vector $Y_{t}$.

First, we compute a "point" forecast for $\mathrm{y}_{t+h}$. The forecast function is given by

$$
\mathrm{y}_{t}(\mathrm{~h})=\mathrm{J}{ }_{r=0}^{\mathrm{r}-1} \mathrm{~B}_{t+h-r} \mathrm{Y}_{t}+{ }_{s=0}^{\mathcal{K}-1} \bigodot_{s t+h} \mathrm{D}_{t+h-s} \mathrm{Z}_{t+h-s}
$$

or, recursively

$$
\mathrm{y}_{t}(\mathrm{~h})=\mathrm{J} \mathrm{B}_{t+h} \mathrm{Y}_{t}(\mathrm{~h} ; \mathrm{1})+\widetilde{\sigma}_{t+h} \mathrm{Z}_{t+h}
$$

where $\widetilde{D}_{t+h}$ is the $\mathrm{NG} f \mathrm{q}$ matrix $\left[\mathrm{d}_{1 t} \mathrm{~d}_{2 t}:: .: \mathrm{d}_{N t}\right]^{\prime}$ and $\mathrm{B}_{t+h}=\operatorname{diag}\left(\mathrm{B}_{1 t} ; \mathrm{B}_{2 t} ;: ; ; \mathrm{B}_{n t}\right)$ with $\mathrm{B}_{i t}=$ $\mathrm{B}_{i t}^{1} ; \mathrm{B}_{i t}^{2} ; ;: ; \mathrm{B}_{i t}^{N}$. One way to obtain a $\mathrm{h}\left\{\right.$ step ahead forecasts is to use the posterior mean of $\mathrm{B}_{t+h}$ and $\widetilde{\sigma}_{t+h}$ and the mean of the predictive density for $\mathrm{z}_{t+h}$, conditional on the information at time 
t. Estimates for the posterior mean of the coe \pm cients can be obtained from the recursive formulas for , $t$ (and, consequently, for ${ }_{t}^{\circ}$ ) using expressions like (9) or by drawing from distributions like (20) and (21) in a recursive fashion. Call this estimates $\hat{\mathrm{B}_{t+h \mid t}}$ and $\hat{\mathrm{D}_{t+h \mid t}}$. The forecast error is $\mathrm{y}_{t+h}$; $\hat{\mathrm{y}_{t}}(\mathrm{~h})={ }_{s=0} \bigodot_{s t+h} \mathrm{u}_{t+h-s}+\left[\mathrm{y}_{t}(\mathrm{~h})\right.$; $\left.\hat{\mathrm{y}_{t}}(\mathrm{~h})\right]$. To measure the forecasting performance it is useful to compute the Mean Square Error (MSE) or the Mean Absolute Error (MAD) of the estimated forecast which are given by

$$
\begin{aligned}
& \operatorname{MSE}\left(\widehat{y_{t}}(\mathrm{~h})\right)={ }_{s=0}^{\mathcal{X}^{-1}} \bigodot_{s t+h} \S_{u} \mathbb{\circlearrowleft}_{s t+h}+\operatorname{MSE}\left[\mathrm{y}_{t}(\mathrm{~h}) \mathrm{i} \hat{y_{t}}(\mathrm{~h})\right] \\
& \operatorname{MAD}\left(\widehat{y_{t}}(\mathrm{~h})\right)={ }_{s=0}^{x-1} j u_{t+h-s} \mathrm{j}+\operatorname{MAD}\left[\mathrm{y}_{t}(\mathrm{~h}) ; \hat{y_{t}}(\mathrm{~h})\right]
\end{aligned}
$$

The ${ }^{-}$rst term on the RHS of each equation can be obtained using posterior mean estimates of $\mathrm{B}_{t+h-r}$ and of $\mathrm{U}_{t}$, conditional on the information at time $\mathrm{t}$, while for the second term an approximation can be computed along the lines of Lätkepohl (1991, p.86\{89). Clearly, if a researcher is interested in point forecasts using the average value of the parameters, then the previous formulas apply using for $\hat{\mathrm{B}}_{t+h \mid t}$ and $\hat{\mathrm{D}}_{t+h \mid t}$ the posteriors derived in section 3.2 .

In many situations, it may be more appealing to compute "average" forecasts $\mathrm{h}\{$ step ahead using the predictive density $\mathrm{f}\left(\mathrm{Y}_{t+h} \mathrm{j} \mathrm{F}_{t}\right)=\mathrm{f}\left(\mathrm{Y}_{t+h} \mathrm{j} \mathrm{F}_{t}\right.$; \# $\mathrm{p}\left(\# \mathrm{j} \mathrm{F}_{t}\right)$ where $\mathrm{f}\left(\mathrm{Y}_{t+h} \mathrm{j} \mathrm{F}_{t}\right.$; \#) is the conditional density of the future observation vector given \#, and $\mathrm{p}\left(\# \mathrm{j} \mathrm{F}_{t}\right)$ is the posterior pdf of \# at timet. To compute forecasts for $\mathrm{Y}_{t+h}$ we can samplefrom the predictive density numerically. For each $\mathrm{i}=1 ;:::_{3} \mathrm{M}$ we draw \# $\#^{(i)}$ from the posterior distribution and simulate the vector $Y_{t+h}^{(i)}$ from the density $\mathrm{f}^{3} \mathrm{Y}_{t+h} \mathrm{j} \mathrm{F}_{t} ; \#^{(i)} . \mathrm{n}_{t+h}^{(i)}{ }_{i=1} \mathrm{O}_{M}$ constitutes a sample, from which we can compute the necessary moments. Thevalue of the forecast is then the ergodic anerage $\hat{Y_{t+h}}=\mathrm{M}^{-1}{ }^{M}{ }_{i=1}^{M} \mathbf{Y}_{t+h}^{(i)}$ and its numerical variance can be estimated using var $\hat{\mathrm{Y}}_{t+h}=\mathrm{M}^{-1} \mathrm{Q}_{o}+{ }_{\mathrm{i}}^{\mathrm{P}_{s=1}} \mathbf{1}_{\mathrm{i}} \frac{s}{r+1} \quad\left(\mathrm{Q}_{s}+\mathrm{Q}_{s}^{\prime}\right)$ where $\mathrm{Q}_{s}=\mathrm{M}^{-1} \mathrm{P}_{i=s+1}^{M} \mathrm{Y}_{t+h}^{(i)}$ i $\hat{\mathrm{Y}}_{t+h} \mathrm{Y}_{t+h}^{(i)} \mathrm{i}{\hat{Y_{t+h}}}_{\mathrm{i}}^{\mathrm{i}}$.

Note that since the computation of the impulse response function for orthogonalized shocks is a simple corollary of the calculation of forecasts, the approach we provide here to calculate point and average forecasts can also be used to compute impulse responses. In fact, given the information up to time $t$, computing impulse response at $t+h$ is equivalent to calculating the di Berence between the conditional forecasts at $t+h$, given that at $t+1$ there has been a one unit impulse in one of the orthogonal shocks, and the unconditional forecast, i.e. with the value of the vector that would have occurred without shocks (see Koop (1992) for an application to structural VAR models). This idea is exploited in a recent paper by Waggoner and Zha (1998). The authors, using a version of (25), develop two bayesian methods for computing probability distributions of conditional forecasts. The last term in (25) represents the dynamic impact of structural shocks which a Bect future realizations of variables through the impulse response matrix $\bigcirc_{s t}$. With conditions or constraints imposed on this last term we can produce what they call conditional forecasts.

In order to compute structural impulse responses and their error bands we must work with a structural VAR, eg. impose some restrictions on the contemporaneous coe \pm cient matrix. A prior 
( ${ }^{\circ}$ at or informative) can then be assigned to the non-zero elements of this matrix, as suggested by Sims and Zha (1998). The extension of their approach to panel date is however not straightforward and we postpone this issue to future work.

Turning point predictions can also be computed from the predictive density of future observations (see in Zellner, Hong and Min (1991)). Let us de- ne turning points as follows:

De $\mathrm{e}^{-}$nition 5.1 A downward turn for unit $\mathrm{i}$ at time $\mathrm{t}+\mathrm{h}+1$ occurs if $\mathrm{S}_{i t+h}$ the growth rate of the reference variable (typically, GNP) satis ${ }^{-}$es for all $\mathrm{h} \mathrm{S}_{i t+h-2} ; \mathrm{S}_{i t+h-1}<\mathrm{S}_{i t+h}>\mathrm{S}_{i t+h+1}$. An upward turn for unit $\mathrm{i}$ at time $\mathrm{t}+\mathrm{h}+1$ occurs if the growth rate of the reference variable satis ${ }^{-}$es $\mathrm{S}_{i t+h-2} ; \mathrm{S}_{i t+h-1}>\mathrm{S}_{i t+h}<\mathrm{S}_{i t+h+1}$.

Similarly, we de ne a non-downward turn and a non-upward turn:

De nition 5.2 A non-downward turn for unit $\mathrm{i}$ at time $\mathrm{t}+\mathrm{h}+1$ occurs if $\mathrm{S}_{i t+h}$ satis ${ }^{-}$es for all $\mathrm{h}$ $\mathrm{S}_{i t+h-2} ; \mathrm{S}_{i t+h-1}<\mathrm{S}_{i t+h} \cdot \mathrm{S}_{i t+h+1}$. A non-upward turn for unit $\mathrm{i}$ at time $\mathrm{t}+\mathrm{h}+1$ occurs if the growth rate of the reference variable satis ${ }^{-}$es $\mathrm{S}_{i t+h-2} ; \mathrm{S}_{i t+h-1}>\mathrm{S}_{i t+h}, \mathrm{~S}_{i t+h+1}$.

Although there are other de nitions in the literature (see eg. Lahiri and Moore (1991)) this is the most used one and it su \pm ces for our purposes. Let $f\left(Y_{i, t+h} j F_{t}\right)={ }_{Y_{p, t+h}} f\left(Y_{t+h} j F_{t}\right) d Y_{p, t+h}$ be the marginal predictivedensit ${ }_{k}$ for the variables of unit $\mathrm{i}$ after integrating the remaining $\mathrm{p}$ variables and let $\mathrm{K}\left(\mathrm{S}_{i t+h}^{1} \mathrm{j}_{t}\right)={ }^{2}:: \mathrm{f}\left(\mathrm{S}_{i t+h}^{1}::: \mathrm{S}_{i t+h}^{G} \mathrm{j}_{t}\right) \mathrm{dS}_{i t+h}^{2}::: \mathrm{dS}_{i t+h}^{G}$ be the marginal predictive density for the growth rate of the reference variable, which we order to be the ${ }^{-}$rst in the list, in unit i.

Take now the simplest case of $\mathrm{h}=0$. To compute the probability of a turning point we have to calculate $S_{i t+1}^{1}$. Given the marginal predictive density $K$, the probability of a downturn in unit $\mathrm{i}$ is

$$
\begin{gathered}
\mathrm{P}_{D t}=\operatorname{Pr}\left(\mathrm{S}_{i t+1}^{1}<\mathrm{S}_{i t}^{1} \mathrm{jS}_{i t-2}^{1} ; \mathrm{S}_{i t-1}^{1}<\mathrm{S}_{i t}^{1} ; \mathrm{F}_{t}\right)= \\
{ }^{\mathrm{Z}_{i t}^{1}} \mathrm{~K}^{3} \mathrm{~S}_{i t+1}^{1} \mathrm{j}_{i t-2}^{1} ; \mathrm{S}_{i t-1}^{1} ; \mathrm{S}_{i t}^{1} ; \mathrm{F}_{t} \mathrm{dS}_{i t}^{1} \\
\quad-\infty
\end{gathered}
$$

and the probability of an upturn is

$$
\begin{aligned}
& \mathrm{P}_{U t} \overline{\bar{Z}}_{\infty} \operatorname{Pr}\left(\mathrm{S}_{i t+1}^{1}>\mathrm{S}_{i t}^{1} \mathrm{j} \mathrm{S}_{i t-2}^{1} ; \mathrm{S}_{i t-1}^{1}>\mathrm{S}_{i t}^{1} ; \mathrm{F}_{t}\right)= \\
& S_{i t}^{1} \\
& \mathrm{~K} \quad \mathrm{~S}_{i t+1}^{1} \mathrm{jS}_{i t-2}^{1} ; \mathrm{S}_{i t-1}^{1} ; \mathrm{S}_{i t}^{1} ; \mathrm{F}_{t} \mathrm{dS_{it } ^ { 1 }}
\end{aligned}
$$

Using a numerical sample from the predictive density satisfying $\mathrm{S}_{i t-2}^{1} ; \mathrm{S}_{i t-1}^{1}<\mathrm{S}_{i t}^{1}$, we can approximate these probabilities using the frequencies of realizations which are less then or greater then $\mathrm{S}_{i t}$. With a symmetric loss function, minimization of the expected loss leads to predict the occurrence of turning point at $\mathrm{t}+1$ if $\mathrm{P}_{D t}>0: 5$ or $\mathrm{P}_{U t}>0: 5$.

For $\mathrm{h} \in 0$ the probability of a turning point can be computed using the joint predictive density for all future observations, i.e. in the case of a downturn,

$$
\begin{aligned}
\mathrm{P}_{D t+h} & =\operatorname{Pr}\left(\mathrm{S}_{i t+h+1}^{1}<\mathrm{S}_{i t+h}^{1}>\mathrm{S}_{i t+h-2}^{1} ; \mathrm{S}_{i t+h-1}^{1} \mathrm{jF}_{t}\right)= \\
\mathrm{Z}_{S_{i t}^{1}} \mathrm{Z}_{\infty} \mathrm{Z}_{\infty} \mathrm{K}^{3} \mathrm{~S}_{i t+h+1}^{1}<S_{i t}^{1} & <\mathrm{S}_{i t+h}^{1}>\mathrm{S}_{i t+h-2}^{1} ; \mathrm{S}_{i t+h-1}^{1} \mathrm{jF}_{t} \mathrm{dS}_{i t+h}^{1} \mathrm{dS}_{i t+h-1}^{1} \mathrm{dS}_{i t+h-2}^{1}
\end{aligned}
$$


Given the available pane data structure we may also be interested in computing the probability that a turning point occurs jointly for $\mathrm{m}$ - $\mathrm{N}$ units of panel. For example, we would like to compute the probability that at $t+1$ there will be a recession in European countries. Let $K\left(S_{t+h}^{1} j F_{t}\right)$ be the joint predictive density of the reference variable for the $m$ units of interest. Then the probability of a downturn is:

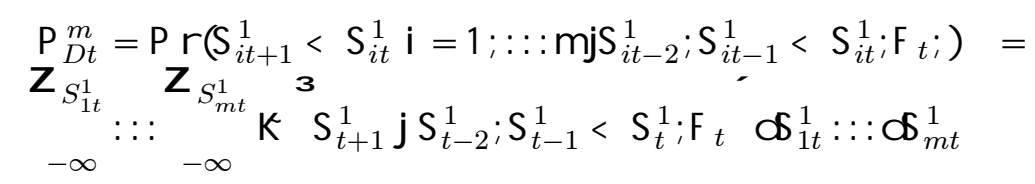

\section{An application}

In this section we apply the methodology to the problem of forecasting growth rates and predicting turning points in the G-7 countries. For each country we consider three national variables (GNP, real stock returns and real money growth) and a world one (the median real stock return in OECD countries) which is assumed to be exogenous in each equation. Hence there are 21 variables in the panel VAR. These variables are chosen after a rough speci cation search over about 10 variables because they appear to have the highest in-sample pairwise and multiple correlation with output growth. Among the variables we tried are the nominal interest rate, the slope of the term structure and in ${ }^{\circ}$ ation. Data is sampled quarterly from 1973,1 to 1993,4 and taken from IMF statistics. Data from 1973,1 to 1988,4 is used to estimate the parameters and data from 1989,1 to 1993,4 to evaluate the forecasting performance and to predict turning points.

We compare the forecasting performance of our pane VAR speci ${ }^{-}$cations with those obtained with other models suggested in the literature. As a benchmark we ${ }^{-}$rst run two versions of a trivariable VAR(2) model for each country separately. The ${ }^{-}$rst one is an unrestricted (VAR). The second a weakly restricted VAR (BVAR) where we use a standard Litterman-prior with a mean of one on the ${ }^{-}$rst lag, a general tightness of 0.15 , no decay in the lags and a weight of 0.5 on the lags of other variables. Since these two models do not exploit cross sectional information nor do they allow for time variation, they can be used as a benchmark to measure the improvements obtained by speci ${ }^{-}$cations which allow any of these two features in the model.

Also for comparison, we run a single equation AR(3) model for GNP growth for each single country, augmented with two lags of real stock returns, 1 lag of real money balances and one lag of the median world real stock return. This is the speci ${ }^{-}$cation used by Garcia Ferrer et al (1987), Zellner and Hong (1989) and Zellner, Hong and Min (1991) to forecast annual growth rates of output in 18 countries. With the extended sample and the higher frequency of the data we have available, we con ${ }^{-} \mathrm{rm}$ their results for all of the G-7 countries. This model represents a restricted version of the previous unrestricted VAR where insigni ${ }^{-}$cant lags are purged from the speci ${ }^{-}$cation. The forecasting power of this model is measured when parameters are estimated with OLS (OLS) and with the three shrinkage procedures: a ridge estimator (RIDGE), an estimator obtained assuming an exchangable prior on the coe \pm cients (as in Garcia Ferrer et al. (1987)) (EXCHANGEABLE) and an estimator obtained using a g-prior (as in Zellner and Hong (1989)) (G-PRIOR). The two latter estimators attempt to improve upon OLS by combining the information coming from each unit 
with the one from the pooled sample. They di ßer in the way they combine individual and pooled information. Notice that none of these estimators allows for time variations in the coe \pm cients.

Finally, as a term of comparison, we use a version of the pane VAR speci ${ }^{-}$cation suggested by Ballabriga et al (1998) (PBVAR). This model speci- cation does not usetheinformation coming from the cross section - every variable is treated in the same way regardless of the country where is from but al lows for time variations in the coe \pm cients of the model. The mode has the same structure as Doan, Litterman and Sims (1984) and assumes that the coe \pm cient vector ${ }^{-}{ }_{t}$ for the entire system has an $\operatorname{AR}(1)$ structure of the form ${ }_{t}{ }_{t}=\mathrm{M}^{-}{ }_{t-1}+\mathrm{u}_{t}$ where $\mathrm{u}_{t}$, conditional on the information available, is normal with mean zero and variance $\S_{u}$. The matrices ${ }^{-}, \mathrm{M}$, and $\S_{u}$ depend on 7 hyperparameters: - ve parameters controlling the structure of $\S_{u_{0}}$ (a general tightness $\left(\mu_{1}\right), a$ tightness on variables of the same country $\left(\mu_{3}\right)$, a tightness on the variables of other countries $\left(\mu_{4}\right)$, a geometric lag decay with parameter $\left(\mu_{2}\right)$, and a tightness on world variables $\left.\left(\mu_{5}\right)\right)$; a parameter describing the structure of $M\left(\mu_{6}\right)$; and a parameter controlling the prior mean on the ${ }^{-r s t}$ lag of ${ }_{0}\left(\mu_{t}\right)$. Table 1 reports the optimal values selected by maximizing the in-sample predictive density of the model with a simplex algorithm.

Weproduceforecasts from two versions of our panel VAR mode: one with a modi ${ }^{-}$ed Minnesotaprior (PANEL1), and one with a fully hierarchical speci ${ }^{-}$cation (PANEL2). In the former, the nine prior parameters are selected to maximize the predictive density using a simplex method. Their optimal values are reported in table2. For both PBVAR and PANEL1 forecasts are computed using the posterior mean of the coe \pm cients, after we have plugged-in the estimates of the prior parameters in the formula. For PANEL2 posterior estimates of the coe \pm cients are computed numerically using MCMC methods and forecasts are directly obtained from these estimates.

In setting up the panel VAR models we assume that $\mathrm{H}=\mathrm{V}$, where $\mathrm{V}$ is known. For the PANEL 1 speci ${ }^{-}$cation we compute the scale factors $V$ and the matrix $\S_{u}$ as follows. We estimatea trivariate VAR for each country and take the average of the estimated variance \{covariance matrix of the residuals across countries as a measure of $\mathrm{V}$. Furthermore, for each of the three variable we estimate a $7\{$ variable VAR (the same variable across countries) and store the variance covariance matrices of the residuals. An estimate of $\S_{u}$ is obtained as:

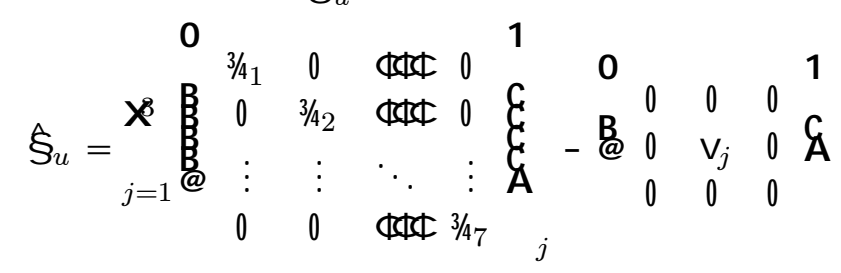

where the ${ }^{-}$rst matrix contains on the diagonal the estimated standard deviations obtained by running the thre 7 vvariate VARs; while the second matrix contains just one element di ßerent from zero, the $(j ; j)$ element, which is obtained from the diagonal of the matrix $V$. For the PANEL2 speci ${ }^{-}$cation we need to choose the scale and the degrees of freedom in the various Wishart distribution. We still set $\mathrm{H}=\mathrm{V}$ with $\mathrm{V}$ estimated as before. Following Kadiyala and Karlsson (1997) we set the degrees of freedom $3 / 8=N+2+(T ; \quad p) a G ; !_{1}=k+2+N+g ; !_{2}=k+2+(t ; p)$ aG while the scale matrices $\mathrm{M}_{0}, \mathrm{~W}_{1}$ and $\mathrm{W}_{2}$ are such that $\S_{u} ; \Varangle ; \S_{\epsilon}$ have the same structure as in the PANEL 1 speci ${ }^{-}$cation. 
We compare the forecasting ability of various models using both the Theil-U Statistics and the Mean Absolute Deviation (MAD) at 1 and 4 periods ahead. These statistics are reported in table 3. Note that the various speci ${ }^{-}$cations we use are in increasing order of complexity and ${ }^{\circ}$ exibility. Therefore, at each stage we can assess the forecasting improvements obtained adding one extra feature to the model.

To examine the performance of various models as business cycle indicators we compute turning points predictions one period ahead. Following Zellner et al. (1991), we compute the total number of turning points, the number of downturns and no-downturns, and the number of upturns and no-upturns in the sample (across all countries) and for each procedure we report the number of correct cases in table 4.

Finally, for each model, we compute the probability that there will be a downward turn in the growth rate of US output in 1989:1-1993:4, given the information available in 1988:4. According to the o \pm cial NBER classi ${ }^{-}$cation the long expansion of the 1980's terminated in 1990:3 and it was followed by a brief and shallow recession. The probabilities for the nine models for each of the 16 periods we consider are presented in table 5.

The forecasting performances of univariate OLS, ridge and exchangeable procedures are very similar. The minimum and maximum values of the Theil-U across countries at one and four steps for the latter two are slightly smaller, but the mean and the median at both steps are practically identical. On the other hand, a univariate model where the parameters are shrunk with a g-prior is somewhat better than OLS in all the dimensions: the maximum, the median, the mean and the minimum value across countries of the Theil-U at both steps are signi ${ }^{-}$cantly lower than those obtained with OLS.

Unrestricted VAR models are not very successful in forecasting growth rates of output, given the large number of parameters to be estimated. This noticeable in particular in the case of J apan, Germany and the UK wherethe Theil-U aresigni ${ }^{-}$cantly worsethan those obtained with univariate speci ${ }^{-}$cations at the one step horizon. However, unrestricted VAR model outperform all univariate speci ${ }^{-}$cations at the four step horizon. Hence, the presence of interdependencies across variables helps in predicting the evolution of the growth rate of output in the medium run. BVAR are signi ${ }^{-}$cantly better than VAR and univariate approaches at the one step horizon. In terms of the median value the gains are of the order of $5-6 \%$ over univariate speci ${ }^{-}$cations and of more than $10 \%$ over the unrestricted VAR. However, the performance at the four step horizon turns out to be inferior to the one of unrestricted VAR, and comparable to the one of univariate shrinkage procedures. This is to be expected since to improve the performance at short horizons BVAR tend to reduce both the memory and the interdependencies of the system, which we have seen are useful exactly when medium-long run forecasts have to be made

Adding time variation in the coe \pm cients and interdependencies across countries substantially improves the forecasting performance both at short and at medium horizons. For example the median Theil-U at one step goes from 0.85 with a simple BVAR to 0.82 with the panel version of this model and for 5 countries the Theil-U is lower by as much as $10 \%$. Similarly, the mean across countries drops by about 3\% with the PBVAR speci ${ }^{-}$cation. The improvement is noticeable also at longer horizons. The distribution of the Theil-U across countries at the four step horizon is similar to the one obtained with a unrestricted VAR, which is the best among the benchmark models. 
Our re- nement of the Litterman's prior, which allow for both cross sectional and time series a-priori restrictions, gives a performance which is essentially similar to the one of the PBVAR model both at the one and at the four step horizons. Few features of the optimally estimated parameters are worth discussing. First, while $\mu_{6}$, the time variation parameter in the variance of is di Berent from zero, it does not appear to add much to the performance of the model. Hence, at least with quarterly data, allowing for heteroskedasticity does not help in improving the quality of the forecasts. Second, while in the PBVAR, the coe \pm cient vector evolves with a persistence of 0.95 but with very small variance, in our PANEL 1 speci ${ }^{-}$cation the time varying component of the coe \pm cients is close to be a white noise. Note that this di Rerence is inconsequential for forecasting and can be explained by examining therole of the parameters regulating thecross sectional prior (i.e. the tightness on $\AA$ and $\circledast$ ). These parameters force a high degree of coherence across countries in the time invariant component and leavethe time varying component to randomly evolve In the PBVAR this distinction is not possible and to produce coetcients which are almost constant over time it is necessary to have close to a random walk dynamics coupled with a small variance Using equation (9), one can see in fact that coetcients of the PANEL1 model areapproximately constant over time and are tightly linked to each other because of the restrictions imposed on ${ }^{\circledR}$. The omission of the - xed erect component, which is precisely what the PBVAR does, biases upward estimates of the persistence parameter and this may explain why the two estimated speci ${ }^{-}$cations are so di Berent. Third, the maximized value of predictive density of the PANEL1 model is signi ${ }^{-}$cantly higher then the one of the PBVAR model (-36.90 vs. -985.35) suggesting that the our speci ${ }^{-}$cation ${ }^{-}$ts the data for the in-sample period better. However, this superior in-sample ${ }^{-} t$ appears to be unimportant for forecasting out-of-sample. That is, the (wrong) restrictions that the PBVAR imposes and which biases the persistence parameter of the time varying coe \pm cients do not translate in poor forecasts at the horizons we consider. We conjecture that this may have to do with the peculiarity of the forecasting sample more than with true similarities between the two speci ${ }^{-}$cations.

The performance of the PANEL2 speci cation is also comparable to the one obtained with PBVAR at the one step horizon. However, while the ranking of the Theil-U across countries in PBVAR and PANEL1 were identical, there is some reshu ${ }^{2}$ ing with the PANEL2 speci ${ }^{-}$cation. That is, the model is somewhat better for J apan and France and somewhat worse for the US and Italy. At the four step horizon the performance of the mode is signi ${ }^{-}$cantly worse than any other mode. While we have not been able to ${ }^{-}$nd a reason for this result, we conjecture that this has to do with the fact that the presence of a large amount of randomness in the speci ${ }^{-}$cation of the model compounds at long horizons and worsens signi ${ }^{-}$cantly its performance In fact, the di ßerence between PANEL 1 and PANEL2 speci cations, apart from problem of precision of estimates is only in the fact that there is an additional layer of uncertainty in the prior of the model.

The relative performance of the various models with the MAD is somewhat similar to the one obtained with the Theil-U at both horizons. However, four features deserve a comment. First, all univariate shrinkage procedures appear to be better than OLS at the one step horizon. The same is true at four steps horizons except for the case of g-prior, which is now signi ${ }^{-}$cantly worse. Second, unrestricted and simple BVAR display a somewhat mediocre performance both at one and four steps horizons. In general, the distribution of the MAD across countries is more concentrated but the mean and the median are above those obtained with univariate shrinkage approaches. Third, 
the improvements obtained with pane VAR approaches are signi ${ }^{-}$cant and our re nement of the Litterman's prior produces thebest distribution of MAD at theonestep horizon. Theimprovements are primarily concentrated for those countries which are in the central part of the distribution and this is re ected in the lower median value we obtain. Fourth, the PANEL2 speci ${ }^{-}$cation is better than any other when we use the mean MAD across country to measure the forecasting speci ${ }^{-}$cations at both horizons. That is, PANEL2 produces a distribution of MAD across countries which is centered below the one obtained with other models and more concentrated. Notice also that the signi $^{-}$cant forecasting di Berences produced by PANEL2 for the Theil-U and the MAD at the four step horizon probably have to do with the di ßerent way the two criteria treat forecasting outliers.

In sum, using interdependencies, adding time variation in the coe \pm cients and using cross sectional restrictions in the prior for the coetcients helps in improving forecasts at short-medium horizon. Nevertheless, it should be pointed out that the distribution of forecasting statistics across countries is very wide, for example, the MAD for I taly is 6 times the one of the US. This di Berences indicate that the process for the growth rate of GDP in some countries does not share much features with the growth rate of GDP of other G-7 countries and that signi ${ }^{-}$cant improvements on the results we present can be obtained by restricting attention to the subset of the countries which are more similar. Also notice that the forecasting performance for US and Canada GDP growth is very similar across speci ${ }^{-}$cations and jointly improves with the complexity of the model, con $^{-}$rming that there are forecasting externalities which can be obtained by cross-sectionally linking the national models for the two countries.

How good are various approaches in predicting turning points? Out of 96 total actual turning points in the sample, univariate approaches recognize between 72 and 75 . Di Berences primarily emerge when we try to predict upturns and non-upturns and for this type of turning points, Zellner's-g approach is better than the others. Unrestricted VAR models are very poor in this dimension and recognize about $10 \%$ less turning points than Zellner's-g approach. The performance of the BVAR model is comparable to the one of univariate Ridge and Exchangeable approadhes but, contrary to them, it predicts upturns and non-upturns better than downturns and non-downturns. The performance of the PBVAR mode is surprisingly poor: it is the second worst in recognizing the total number of turning points and is comparable to unrestricted VARs in predicting downturns and non-downturns. Finally, our two Panel approaches produce 73 and 74 turning point forecasts and recognize the same number of upturns and non-upturns. Comparatively speaking, they substantially improve over PBVAR and are competitive with the best approaches.

Three further conclusions can be drawn from table 4. First, di eerent models are better in recognizing di ßerent types of turning points. If predicting downturns (and non-downturns) is more important than predicting upturns (and non-upturns) our results suggest that VAR, BVAR and PBVAR should not be used. Second, while in terms of linear forecasting statistics there was a clear ranking of procedures, with more complicated ones doing a better job, when we look at nonlinear forecasting statistics, simple univariate approaches, and OLS in particular, are as good as other more re ned approaches. Third, Panel VAR models of the type we have proposed do a better job than any other procedure when we jointly use linear and nonlinear statistics to measure forecasting performance.

Given that our suggested speci ${ }^{-}$cations are good in forecasting on average, we would like to 
know if they are also good in predicting a speci ${ }^{-}$c episode of interest, i.e, the downward turn in real activity occurred in the US in 1990:3. This is interesting because alternative approaches, which were forecasting pretty well in the sample 1970-1980, failed to ${ }^{-}$nd any relevant signs in the data that would predict that a downturn and a short recession were forthcoming (see eg. Stock and Watson (1993)). Interestingly enough, and contrary to most forecasting models, all procedures predict that there is a signi ${ }^{-}$cant probability that a peak in economic activity will occur at 1990:3. For univariate procedures this probability is much larger than the threshold of 0.5 which we use to consider the date a downward turn. In fact all four univariate approaches predict the existence of a peak with probability above 0.64 . Single country VAR, with and without a Bayesian prior are worse than univariate procedures (probability 0.32 and 0.36 respectively) but this may be due to the larger number of parameters to be estimated with the information available at 1988:4. The PBVAR speci ${ }^{-}$cation is overwhelmingly predicting a downward turn in 1990:3 (probability is 0.82) and does not produce any false alarm in the neighborhood of this date. The second Panel VAR approach improves over single country VAR substantially and a produce probability of a downturn in 1990:3 which is comparable with those of univariate approaches. The performance of the ${ }^{-}$rst Panel approach is poor and fails to produce a probability in excess of 0.5 in 1990:3. Note also that while univariate approaches have the tendency to produce a false alarm in 1989:4, probably due to the stock market crash of the fall of 1989, the probabilities produced by VAR and BVAR at dates other than 1990:3 are small and never exceed 0.5. The PBVAR model, on the other hand, produces a high probability of a downturn in 1991:3 a date wherea downturn materialized. The second pane speci ${ }^{-}$cation also produces a high probability of a downward turn in 1991:3 while the probabilities at other dates are small. Finally notice that the peak in 1989:2 is missed by all approaches: the ones which give highest probability to this event are the PBVAR (0.42) and the ${ }^{-}$rst Pane VAR approach (0.41).

In conclusion, our proposed Bayesian PANEL VAR approach is at least as good as any other approach we have examined and in many cases improves the forecasting performance of existing speci ${ }^{-}$cation. This is true when we compare procedures using linear and non-linear forecasting statistics and when we look at speci ${ }^{-} \mathrm{c}$ historical episodes.

\section{Conclusions}

The task of this paper was to describe the issues of speci cation, estimation and forecasting in a macro-pane VAR model with interdependencies. The point of view used is Bayesian. Such an approach has been widely used in the VAR literature since the works of Doan, Litterman and Sims (1984), Litterman (1986), and Sims and Zha (1998) and provides a convenient framework where one can allow for both interdependencies and meaningful time variations in the coetcients. We decompose the parameter vector into two components, one which is unit speci ${ }^{-} \mathrm{c}$ and the other which is time speci ${ }^{-} \mathrm{c}$. We specify $a{ }^{\circ}$ exible prior on these two components which parsimoniously takes into account possible interdependencies in the cross section and allows for time variations in the evolution of the parameters over time. The prior shares features with those of Lindlay and Smith (1972), Doan, Litterman and Sims (1984) and Hsiao et al. (1998) and it is speci ${ }^{-}$ed to havea hierarchical structure, which allows for various degrees of ignorance in the researcher's information 
about the parameters.

Bayesian VARs are known produce better forecasts than unrestricted VAR and, in many situations, ARIMA or structural models (Canova (1995) for references). By allowing interdependencies and some degree of information pooling across units in the model speci cation we introduce an additional level of ${ }^{\circ}$ exibility which may improve the forecasting ability of these models.

We analyze several special cases of our speci cation and compute Bayesian estimators for the mean parameter in the cross section and for the individual coe \pm cients. In some cases analytical formulas for the posterior mean are available using standard formulas. Whenever the parameters of the prior are unknown, we employ the predictive density of the model to estimate them and plug-in our estimates in the relevant formulas in an empirical Bayes fashion.

In the case of fully hierarchical priors, a Markov Chain Monte Carlo method (theGibbs sampler) is employed to calculate posterior distributions. Such an approach is particularly useful in our setup since it exploits the recursive features of the posterior distribution. Recursive formulas for multistep, multiunit forecasts, consistent with the information available at each point in time, are provided using the posterior of the parameters or the predictive density of future observations. The predictive density of future observation is also used to compute turning point probabilities.

To illustrate the performance of the proposed approach, we apply the methodology to the problem of predicting output growth, of forecasting turning points in output growth and computing the probability of a recession in the G-7 using a thre variables (output growth, real stodk returns and real money growth) for each country in the pane. To evaluate the model we also provide a forecasting comparison with other speci ${ }^{-}$cations suggested in the literature We show that our panel VAR approach improves over existing univariate and simple BVAR models when we measure the forecasting performance using the Theil-U and the MAD criteria both at the one step and at the four steps horizons. The improvements are of the order of $5-10 \%$ with the Theil-U and about $2-4 \%$ with the MAD. The forecasting performance of our speci ${ }^{-}$cation is also slightly better then the one of a BVAR model which mechanically extends the Litterman prior to the panel case. In terms of turning point prediction, the two versions of our panel approach are able to recognize about $80 \%$ of turning points in the sample and they turn out to be the best for this task, along with Zellner's g-prior shrinkage approach. The simple extension of the Litterman's prior to the pane case does poorly along this dimension and, among all the procedures employed is the second worst. Finally, all the procedures produce a high probability of a downturn at 90:3, the date selected by the NBER committee to terminate the long expansion of the 80 's. In this instance, our approach is competitive with the best and avoids the false alarms that other approaches produce at other dates.

We consider the work presented in this paper as the ${ }^{-}$rst step in developing a coherent theory for Bayesian Panel VAR models which take into consideration both the nature of interdependencies, the similarities in the statistical mode across units and the existence of time variation in the coe \pm cients. Extensions of the theory outlined here include the formulation of interesting hypothesis on the nature of the interdependencies, on the similarities across units and on time variations and the development of tools to undertake structural identi ${ }^{-}$cation in these models. The work of Sims and Zha (1998) is the starting point for extensions in this latter case 
Proof of proposition 1

Appendix

(i) Notice that (14) and (15) can be written as

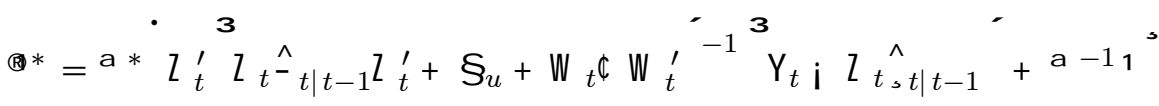

$$
\begin{aligned}
& \underline{\mathrm{a}} *=\underline{\mathrm{a}}^{-1}+\mathrm{Z}_{t}^{\prime} \mathrm{Z}_{t} \hat{-}_{t \mid t-1} \mathrm{Z}_{t}^{\prime}+\S_{u}+\mathrm{W}_{t} \Phi \mathrm{W}_{t}^{\prime}{ }^{\prime-1} \mathrm{Z}_{t}{ }^{-1}
\end{aligned}
$$

Setting ${ }^{\mathrm{a}}-1=0$, the result follows.

(ii) The posterior distribution of ®is normal with mean ${ }^{*}=\mathrm{S}_{N}{ }^{1}+\dot{\mathrm{A}}_{12} \dot{\mathrm{A}}_{22}^{-1}\left[\mathrm{Y}_{t} \mathbf{i} \mathrm{Z}_{t}\left({ }^{1}+{ }_{, t \mid t-1}\right)\right]$ and variance $\mathrm{V}_{\alpha}^{*}=\hat{A}_{11}$ i $\hat{A}_{12} \hat{A}_{22}^{-1} \hat{A}_{21}$ where $\hat{A}_{11}=\left(\mathrm{S}_{N}\right.$ a $\left.\mathrm{S}_{N}^{\prime}+\phi\right) ; \hat{A}_{12}=\left(\mathrm{S}_{N}\right.$ a $\left.\mathrm{S}_{N}^{\prime}+థ\right) \mathrm{W}_{t}^{\prime} ; \hat{A}_{21}=$

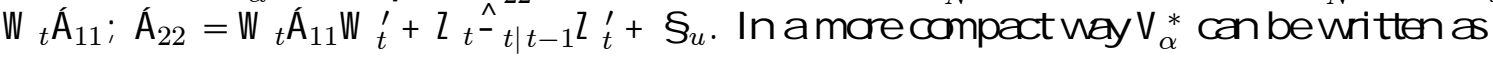

$$
\mathrm{V}_{\alpha}^{*}=\mathrm{W}_{t}^{\prime} \S_{u}+\mathrm{Z}_{t^{-}{ }{ }_{t \mid t-1}} \mathrm{Z}_{t}^{\prime}{ }^{-1} \mathrm{~W}_{t}+{ }^{\mathrm{i}} \mathrm{S}_{N} \mathrm{a}_{N}^{\prime}+\phi^{\phi_{-1}{ }^{-1}}:
$$

Notice that $\left(\mathrm{S}_{N} \underline{\underline{a}} \mathrm{~S}_{N}^{\prime}+\pitchfork\right)^{-1}=\Phi^{-1} \mathrm{i} \phi^{-1} \mathrm{~S}_{N}{ }^{\mathrm{i}} \mathrm{S}_{N}^{\prime} \phi^{-1} \mathrm{~S}_{N}+\underline{a}^{-1}{ }^{\phi}-1 \mathrm{~S}_{N}^{\prime} \phi^{-1}$. Hence, for $\underline{\mathrm{a}}-1=0$, this expression is equal to $\mathrm{F}$. Moreover the posterior mean can be written as

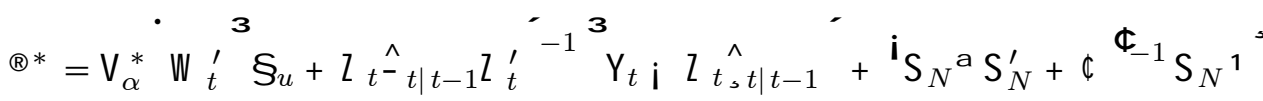

Using the previous result and the fact that $\mathrm{FS}_{N}=0$, the result follows.

(iii) The posterior mean and variance of,$t$ can be written as

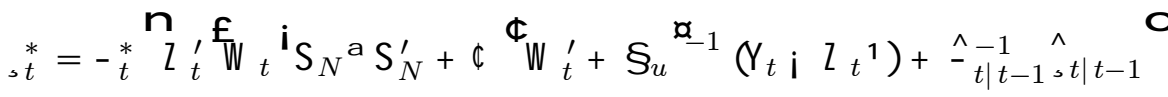

$$
\begin{aligned}
& -{ }_{t}^{*-1}=\mathrm{Z}_{t}^{\prime}{ }^{\mathrm{f}} \mathrm{W}_{t}{ }^{\mathrm{i}} \mathrm{S}_{N} \underline{\underline{a}} \mathrm{~S}_{N}^{\prime}+\phi{ }^{\Phi} \mathrm{W}_{t}^{\prime}+\S_{u}{ }^{\mathrm{\alpha}_{-1}} \mathrm{Z}_{t}+\hat{-}_{t \mid t-1}^{-1}
\end{aligned}
$$

The matrix $\mathrm{W}_{t}\left(\mathrm{~S}_{N}{ }^{\mathrm{a}} \mathrm{S}_{N}^{\prime}+\phi\right) \mathrm{W}_{t}^{\prime}+\S_{u}$ can be written as $Z_{t}{ }^{\mathrm{a}} \mathrm{Z}_{t}^{\prime}+\left(\mathrm{W}_{t} \phi \mathrm{W}_{t}^{\prime}+\S_{u}\right)$ and its inverse is equal to $M^{-1}$ i $M^{-1} Z_{t}{ }^{i} Z_{t}^{\prime} M^{-1} Z_{t} \hbar^{a-1}{ }^{\Phi_{-1}} Z_{t}^{\prime} M^{-1}$ where $M=\left(W_{t} \notin W_{t}^{\prime}+\S_{u}\right)$. Setting ${ }^{-1}=$ 0 ; the last expression reduces to $M^{-1} \mathrm{I}_{\mathrm{i}} \mathrm{Z}_{t}{ }^{\mathrm{i}} \mathrm{Z}_{t}^{\prime} \mathrm{M}^{-1} \mathrm{Z}_{t}{ }^{4} \mathrm{Z}_{t}^{\prime} \mathrm{M}^{-1}$. Premultiplying this matrix by $Z_{t}^{\prime}$, we get a zero matrix. Hence, from (35) and (36) $-{ }_{t}^{*-1}=\hat{-}_{t \mid t-1}^{-1},{ }_{t}^{*}=\hat{s} \hat{s}_{t-1}$ and the posterior distribution for ${ }_{t}$ is just equal to the prior. 
Proof of proposition 2

Recall that $\S_{\varepsilon}^{-1}=0$ implies $\widehat{\widehat{-}_{t \mid t-1}^{-1}}=0$ :

(i) Çonsider the posterior moments (31) and (32). $\left[\left(\mathrm{W}_{t} \nsubseteq \mathrm{W}_{t}^{\prime}+\S_{u}\right)+\mathrm{Z}_{t^{-}} \hat{}_{t \mid t-1} \mathrm{Z}_{t}^{\prime}\right]^{-1}=\mathrm{M}^{-1} \mathbf{i}$ $M^{-1} Z_{t} Z_{t}^{\prime} M^{-1} Z_{t}+\hat{-}_{t \mid t-1}^{-1}{ }^{-1} Z_{t}^{\prime} M^{-1}$ where $M$ was previously de ned. When $\hat{-}_{t \mid t-1}^{-1}=0$; this reduces to $M^{-1}$ i $M^{-1} Z_{t}{ }^{i} Z_{t}^{\prime} M^{-1} Z_{t}{ }^{\Phi}-1 Z_{t}^{\prime} M^{-1}$ which gives a zero matrix if premultiplied by $Z_{t}^{\prime}$. Consequently $\underline{a} *=\underline{a}{ }^{1}$ and the posterior distribution of is just equal to its prior.

(ii) Consider the posterior moments (33) and (34). When $\hat{-}_{t \mid t-1}^{-1}=0, \S_{u}+Z_{t^{-}} \hat{}_{t \mid t-1} Z_{t}^{\prime}{ }^{-1}=$ $\mathrm{S}=\S_{u}^{-1} \mathbf{i} \S_{u}^{-1} \mathbf{Z}_{t}{ }^{i} Z_{t}^{\prime} \S_{u}^{-1} \mathbf{Z}_{t}{ }^{{ }^{-1}} \mathbf{Z}_{t}^{\prime} \S_{u}^{-1}$. Substituting into (33) and (34), gives the result.

(iii) The proof of this statement comes straight from (35) and (36).

$\mathrm{De}^{-}$nition of the matrices for the Gibbs sampler

$$
\begin{aligned}
& \widehat{\mathrm{W}}_{1}=\mathrm{W}_{1}+{ }^{\mathrm{X}}{ }^{\mathrm{i}} \mathrm{A}_{i} \mathrm{E}_{i} ; \mathrm{A}^{2} \mathrm{\Phi}^{1 /} \mathrm{V}^{-1}{ }^{\mathrm{i}} \mathrm{A}_{i} \mathrm{E}_{i} ; \mathrm{A}^{2} ; \\
& \widehat{W}_{2}=\mathrm{W}_{2}+{ }^{i}\left(\boldsymbol{\alpha}_{t} \mathrm{i}^{1 / \alpha_{t-1}}\right)^{\prime} \mathrm{V}_{1}^{-1}\left(\boldsymbol{\alpha}_{t} \mathrm{i}^{1 / \alpha_{t-1}}\right)
\end{aligned}
$$

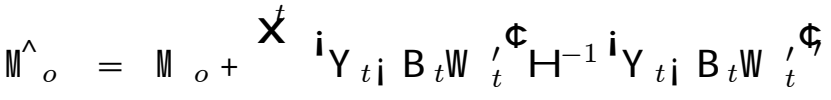

$$
\begin{aligned}
& \widehat{\mathrm{P}_{o}}=\mathrm{P}_{o}+{ }^{\mathrm{X}^{\prime}}{ }^{\mathrm{i}} \mathrm{Y}_{t} \mathrm{i} \mathrm{B}_{t} \mathrm{~W}_{t}^{\prime \Phi_{1}} \S^{-1}{ }^{\mathrm{i}} \mathrm{Y}_{t} \mathrm{i} \mathrm{B}_{t} \mathrm{~W}_{t}^{\prime{ }^{\phi}} \\
& \tilde{\mathrm{A}}_{\mathrm{X}}{ }^{t} \text { ! } \\
& \text { (B) }={\widehat{V_{\alpha}}}^{X} W_{t}^{\prime}(\S-H)^{-1}\left(Y_{t} ; Z_{t, t}\right)+\Phi^{-1} S_{N} \circledast
\end{aligned}
$$

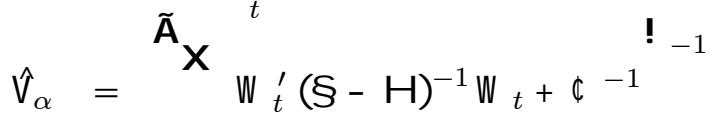

$$
\begin{aligned}
& \Re^{*}=\widehat{\mathrm{V}}^{*}\left(\mathrm{~V}--_{1}\right)^{-1} \mathrm{X} \quad \mathrm{R}_{i} \AA_{i}+\underline{\mathrm{a}}-11 \\
& \hat{\mathrm{V}}^{*}={ }^{3} \mathrm{~N}\left(\mathrm{~V}--_{1}\right)^{-1}+\underline{a}^{i}{ }^{\prime}-1
\end{aligned}
$$

where $Y_{t}$ is $\mathrm{N} £ \mathrm{G}, \mathrm{B}_{t}$ is $\mathrm{N} £ \mathrm{Gk}$ and $\mathrm{W}_{t}=\left(\mathrm{I}_{G}-\mathrm{X}_{t}^{\prime}\right)$ : Model (1) is just a raw vectorization of $\mathrm{Y}_{t}=\mathrm{B}_{t} \mathrm{~W}_{t}^{\prime}+\mathrm{U}_{t}$, where $\mathrm{B}_{t}=\left[\operatorname{vecr}\left(\mathrm{B}_{1 t}\right) ; ; \ldots ; \text { vecr }\left(\mathrm{B}_{N t}\right)\right]^{\prime}$ : Here vecr () is the row vectorization of a matrix; $\mathrm{B}_{i t}=\mathrm{A}_{i}+\alpha_{t} \mathrm{E}_{i}$ is a $\mathrm{G} \mathrm{f}$ matrix and the parameter vectors $\mathbb{R}_{l}$ and,$t$ in (4) and (6) are the row vectorizations of $A_{i}$ and $\alpha_{t}$ respectively. 


\section{References}

[1] Anderson B.D.O., J .B. Moore (1979), Optimal Filtering, Prentice\{Hall.

[2] Ballabriga F. C., M. Sebastian and J . Vall (\$S (1998), European Asymmetries, forthcoming, J ournal of International Economics.

[3] Berger J .O. (1985), Statistical Decision Theory and Bayesian Analysis, New York: Springer-Verlag, 2nd ed.

[4] Canova, F. (1995), VAR: Speci cation, Estimation, Testing, Forecasting in H. Pesaran and M. Wickens (eds.) Handbook of Applied Econometrics, Blackwell Press.

[5] Canova, F. and A. Marcet (1997), The poor stay poor: non \{convergence across countries and regions, forthcoming, Review of Economic Studies.

[6] Carter, C.K and R.Kohn (1994), On Gibbs sampling for state space models, Biometrika , 81(3):541\{ 553.

[7] Chamberlain, G. (1982), Multivariate regression model for panel data, J ournal of Econometrics, 18:5\{ 46.

[8] Chamberlain, G. (1984), Pane data in Handbook of Econometrics II , Z. Griliches and M.D.Intriligator (eds.), Amsterdam North Holland, $1247\{1318$.

[9] Chib, S. and E. Greenberg (1996), Markov chain Monte Carlo simulation methods in econometrics, Econometric Theory, 12:409\{431.

[10] Doan, T., R. Litterman and C. Sims (1984), Forecasting and conditional projections using realist prior distributions, Econometric Review, 3(1), 1\{100.

[11] Garcia Ferrer, A, High ${ }^{-}$eld, F., Palm, F. and Zellner, A. (1987), Macroeconomic forecasting using pooled international data, J ournal of Business and Economic Statistics, 5, 53-67.

[12] Gelfand A.E. and A.F.M. Smith (1990), Sampling\{based approaches to calculating marginal densities, J ournal of the American Statistical Association, 85(410):398\{409.

[13] Geman S. and D. Geman (1984), Stochastic relaxation, Gibbs ditributions and the Bayesian restoration of images, IEEE Transactions on Pattern Analysis and Machine Intelligence, 6(6):721 $\{741$.

[14] Gerlach, S. and F. Smets (1996), The monetary transmission mechanism: evidence from the G\{7 countries, mimeo, Bank of International Settlements, Basle.

[15] Ho®maister, W. and J .F.Rold\$s (1997), Are business cycles di ßerent in Asia and Latin America?, IMF working paper no. $97 / 79$.

[16] Holtz\{Eakin D., W. Newey and H. Rosen (1988), Estimating vector autoregressions with panel data, Econometrica, 56(6) pp. $1371\{1395$. 
[17] Hsiao, C., M.H. Pesaran and A.K. Tahmiscioglu (1997), Bayes estimation of short run coetcients in dynamic panel data models, mimeo, Cambridge University.

[18] Kadiyala K.R. and S. Karlsson (1997), Numerical methods for estimation and inference in Bayesian VAR models, J ournal of Applied Econometrics, 12:99\{132.

[19] Koop, G (1992), Aggregate shocks and macroeconomic ${ }^{\circ}$ uctuations: a Bayesian approach, J ournal of Applied Econometrics, 7:395\{411.

[20] Ingram, B. and Whiteman, C. (1994), "Supplanting the Minnesota Prior: Forecasting Macroeconomic Series using Real Business Cycles Model Prior", J ournal of Monetary Economics , 34, 497-510.

[21] Lahari, K. and Moore J . (1991) Leading Economic Indicators: New Approaches and Forecasting Records, Chicago, IL: University of Chicago Press.

[22] Lindley, D.V. and A.F.M. Smith (1972), Bayes estimates for thelinear model (with discussion), J ournal of the Royal Statistical Society, B, 34, pp. $1\{41$.

[23] Litterman, R.B. (1986), Forecasting with Bayesian Vector Autoregressions\{Five years of experience, J ournal of Business and Economic Statistics, 4:25\{38.

[24] Lätkepohl, H. (1990), Introduction to Multiple Time Series Analysis, Berlin: Springer \{Verlag.

[25] Pesaran M.H. and R.Smith (1996), Estimating long run relationships from dynamic heterogeneous panels, J ournal of Econometrics, 68:79\{113.

[26] Rebucci, A (1998), External shocks, macroeconomic policy and growth: a panel VAR approach, Global Economic Institutions, working paper 40.

[27] Sims, C. and T.Zha (1998), Bayesian methods for dynamic multivariatemodels, I nternational Economic Review , 39, 949-979.

[28] Stock, J . and Watson, M. (1993) Predicting Recessions in Stock, J . and M. Watson. (eds) New Research on Business Cycles, Indicators and Forecasting, Chicago, II.: Chicago University Press.

[29] Waggoner, D.F. and T.Zha (1998), Conditional Forecasts in Dynamic Multivariate Models, Federal Reserve Bank of Atlanta, Working Paper n. $98\{22$.

[30] Zellner, A. (1971), An Introduction to Bayesian Inference in Econometrics, New York: Wiley.

[31] Zellner, A. and Hong, C (1989), Forecasting International Growth Rates Using Bayesian Shrinkage and other procedures, J ournal of Econometrics, 40, 183-202.

[32] Zellner, A., Hong, C. and Min, C. (1991), Forecasting turning point in international output growth rates using Bayesian exponentially weighted autoregression, time varying parameter, and, pooling techniques, J ournal of Econometrics, 49, 275-304. 
Tables

Table 1: Estimated Hyperparameters: PBVAR

\begin{tabular}{|ll|}
\hline General tightness $\left(\mu_{4}\right)$ & 0.01 \\
\hline Lag decay $\left(\mu_{2}\right)$ & 13.96 \\
\hline Own country tightness $\left(\mu_{3}\right)$ & $3.5-\mathrm{e} 005$ \\
\hline Other countries tightness $\left(\mu_{4}\right)$ & $7.3-\mathrm{e} 004$ \\
\hline World variable tightness $\left(\mu_{5}\right)$ & $5.0 \mathrm{e}-007$ \\
\hline AR coe \pm cient $\left(\mu_{6}\right)$ & 0.95 \\
\hline Prior mean on the ${ }^{-}$rst lag $\left(\mu_{7}\right)$ & 0.11048 \\
\hline
\end{tabular}

Table 2: Estimated Hyperparameters: PANEL1

\begin{tabular}{|ll|}
\hline Tightness for ${ }^{\circledR}\left(\mu_{1 \alpha}\right)$ & 0.1207 \\
\hline Tightness for,$\left(\mu_{1 \lambda}\right)$ & 0.1300 \\
\hline Tightness for $\circledast^{\circledR}\left(\mu_{\bar{\alpha}}\right)$ & 0.0004 \\
\hline Lag decay $\left(\mu_{2}\right)$ & 1.9156 \\
\hline Tightness on other countries $\left(\mu_{8}\right)$ & 0.0046 \\
\hline Tightness on world variables $\left(\mu_{4}\right)$ & 4.7804 \\
\hline Law of motion of , $\left(\mu_{5}\right)$ & 0.1211 \\
\hline Time variation $\left(\mu_{6}\right)$ & 0.4295 \\
\hline Prior mean on ${ }^{-}$rst lag $\left(\mu_{7}\right)$ & 0.0754 \\
\hline
\end{tabular}


Tables

Table 3

Theil-U Statistics

\begin{tabular}{|c|c|c|c|c|c|c|c|c|c|c|}
\hline Method & Step & US & apan & Germany & UK & France & Italy & Canada & Median & Mean \\
\hline \multirow[t]{2}{*}{ VAR } & 1 & 1.06 & 0.88 & 0.91 & 0.94 & 1.00 & 0.73 & 0.95 & 0.94 & 0.92 \\
\hline & 4 & 0.73 & 0.95 & 0.56 & 0.81 & 1.32 & 0.96 & 0.72 & 0.81 & 0.86 \\
\hline \multirow[t]{2}{*}{ BVAR } & 1 & 0.83 & 0.89 & 0.69 & 0.91 & 0.90 & 0.80 & 0.85 & 0.85 & 0.84 \\
\hline & & 0.75 & 0.89 & 0.65 & 0.79 & 1.16 & 1.00 & 0.70 & 0.89 & 0.85 \\
\hline \multirow[t]{2}{*}{ OLS } & 1 & 1.21 & 0.86 & 0.88 & 0.86 & 0.90 & 0.79 & 0.91 & 0.88 & 0.90 \\
\hline & 4 & 0.77 & 0.90 & 1.07 & 0.76 & 0.98 & 1.03 & 0.67 & 0.90 & 0.88 \\
\hline \multirow[t]{2}{*}{ Ridge } & 1 & 1.17 & 0.83 & 0.89 & 0.85 & 0.89 & 0.79 & 0.89 & 0.89 & 0.90 \\
\hline & 4 & 0.76 & 0.88 & 1.06 & 0.75 & 0.99 & 1.01 & 0.68 & 0.88 & 0.87 \\
\hline \multirow[t]{2}{*}{ Exchangeable } & 1 & 1.18 & 0.84 & 0.90 & 0.85 & 0.89 & 0.78 & 0.89 & 0.89 & 0.90 \\
\hline & 4 & 0.76 & 0.90 & 1.09 & 0.75 & 0.99 & 1.01 & 0.68 & 0.90 & 0.88 \\
\hline \multirow[t]{2}{*}{ g-prior } & 1 & 1.06 & 0.86 & 0.69 & 0.78 & 1.00 & 0.72 & 0.92 & 0.86 & 0.86 \\
\hline & 4 & 0.83 & 1.07 & 0.77 & 0.75 & 1.12 & 1.02 & 0.70 & 0.83 & 0.89 \\
\hline \multirow[t]{2}{*}{ PBVAR } & 1 & 0.82 & 0.85 & 0.68 & 0.76 & 0.98 & 0.73 & 0.85 & 0.82 & 0.81 \\
\hline & 4 & 0.86 & 0.91 & 0.77 & 0.75 & 1.08 & 1.03 & 0.66 & 0.86 & 0.87 \\
\hline \multirow[t]{2}{*}{ Panel 1} & 1 & 0.81 & 0.88 & 0.67 & 0.75 & 1.02 & 0.70 & 0.88 & 0.81 & 0.81 \\
\hline & 4 & 0.86 & 0.90 & 0.76 & 0.74 & 1.07 & 1.03 & 0.66 & 0.86 & 0.86 \\
\hline \multirow[t]{2}{*}{ Panel 2} & 1 & 0.93 & 0.81 & 0.69 & 0.78 & 0.99 & 0.78 & 0.85 & 0.81 & 0.82 \\
\hline & 4 & 0.83 & 1.59 & 1.62 & 1.55 & 1.47 & 1.93 & 0.90 & 1.55 & 1.41 \\
\hline \multicolumn{11}{|c|}{ MAD Statistics } \\
\hline \multirow[t]{2}{*}{ VAR } & 1 & 0.46 & 1.71 & 1.74 & 1.35 & 1.26 & 2.91 & 0.65 & 1.35 & 1.44 \\
\hline & 4 & 0.35 & 1.55 & 1.18 & 1.33 & 1.66 & 2.74 & 0.56 & 1.33 & 1.34 \\
\hline \multirow[t]{2}{*}{ BVAR } & 1 & 0.46 & 1.62 & 1.48 & 1.32 & 1.15 & 3.22 & 0.58 & 1.32 & 1.40 \\
\hline & 4 & 0.40 & 1.39 & 1.25 & 1.28 & 1.42 & 2.98 & 0.51 & 1.28 & 1.40 \\
\hline \multirow[t]{2}{*}{ OLS } & 1 & 0.56 & 1.59 & 1.51 & 1.37 & 1.06 & 3.17 & 0.57 & 1.37 & 1.40 \\
\hline & 4 & 0.34 & 1.54 & 1.58 & 1.28 & 1.14 & 3.19 & 0.54 & 1.28 & 1.37 \\
\hline \multirow[t]{2}{*}{ Ridge } & 1 & 0.54 & 1.50 & 1.68 & 1.31 & 1.07 & 3.14 & 0.56 & 1.31 & 1.40 \\
\hline & 4 & 0.36 & 1.46 & 1.72 & 1.25 & 1.17 & 3.09 & 0.53 & 1.25 & 1.37 \\
\hline \multirow[t]{2}{*}{ Exchangeable } & 1 & 0.54 & 1.52 & 1.68 & 1.32 & 1.06 & 3.14 & 0.56 & 1.32 & 1.40 \\
\hline & 4 & 0.35 & 1.48 & 1.73 & 1.26 & 1.17 & 3.09 & 0.53 & 1.26 & 1.37 \\
\hline \multirow[t]{2}{*}{ g-prior } & 1 & 0.53 & 1.63 & 1.33 & 1.18 & 1.26 & 2.89 & 0.54 & 1.26 & 1.34 \\
\hline & 4 & 0.41 & 1.60 & 1.35 & 1.18 & 1.34 & 3.12 & 0.51 & 1.34 & 1.36 \\
\hline \multirow[t]{2}{*}{ PBVAR } & 1 & 0.46 & 1.47 & 1.29 & 1.17 & 1.27 & 2.85 & 0.53 & 1.27 & 1.29 \\
\hline & 4 & 0.44 & 1.48 & 1.27 & 1.12 & 1.31 & 3.14 & 0.51 & 1.27 & 1.32 \\
\hline \multirow[t]{2}{*}{ Panel 1} & 1 & 0.46 & 1.53 & 1.24 & 1.08 & 1.37 & 2.82 & 0.54 & 1.24 & 1.29 \\
\hline & 4 & 0.44 & 1.48 & 1.27 & 1.11 & 1.31 & 3.14 & 0.50 & 1.27 & 1.32 \\
\hline \multirow[t]{2}{*}{ Panel 2} & 1 & 0.49 & 1.45 & 1.27 & 1.18 & 1.32 & 3.09 & 0.60 & 1.27 & 1.34 \\
\hline & 4 & 0.55 & 1.40 & 1.25 & 1.11 & 1.43 & 2.96 & 0.65 & 1.25 & 1.33 \\
\hline
\end{tabular}

Notes: VAR is a VAR(2) model for output growth, real stock returns and real money growth, BVAR is the same model with a Minnesota prior. OLS refer to a model where the parameters are estimated with OLS, Ridge to a Ridge correction, Exchangeable to a model with an excheangeable prior and g-prior to Zellner's g-prior speci cation. PBVAR is a 21 VAR model with a Minnesota prior and time variations, Pane 1 is a panel VAR model with all 7 countries with a modi ${ }^{-}$ed Minnesota prior and Pane 2 is the same model with a hierarchical prior. 
Tables

Table 4: Turning points forecasts

\begin{tabular}{llcc}
\hline \hline Method & Turning Points & DT \& NDT & UT \& NUT \\
\hline TRUE & 96 & 47 & 49 \\
\hline VAR & 65 & 32 & 33 \\
BVAR & 72 & 34 & 38 \\
\hline OLS & 74 & 37 & 37 \\
Ridge & 72 & 37 & 35 \\
Exchangeable & 72 & 37 & 35 \\
g-prior & 75 & 37 & 38 \\
\hline PBVAR & 68 & 32 & 36 \\
\hline Panel 1 & 73 & 36 & 37 \\
Panel 2 & 74 & 37 & 37 \\
\hline
\end{tabular}

Notes: VAR is a VAR(2) model for output growth, real stock returns and real money growth, BVAR is the same model with a Minnesota prior. OLS refer to a model where the parameters are estimated with OLS, Ridge to a Ridge correction, Exchangeable to a model with an excheangeable prior and g-prior to Zellner's g-prior speci ${ }^{-}$cation. PBVAR is a 21 VAR model with a Minnesota prior and time variations Panel 1 is a panel VAR mode with all 7 countries with a modi ${ }^{-}$ed Minnesota prior and Pand 2 is the same model with a hierarchical prior. DT means downturn, NDT means non-downturn, UT means upturn and NUT means a non-upturn.

Table 5: Probabilities of a downturn in US GDP growth

\begin{tabular}{lccccccccc}
\hline \hline quarter & VAR & BVAR & OLS & RIDGE & EXCHANGEABLE & g-PRIOR & PBVAR & PANEL1 & PANEL2 \\
\hline $89: 1$ & 0.000 & 0.000 & 0.000 & 0.000 & 0.000 & 0.000 & 0.000 & 0.000 & 0.000 \\
$89: 2^{\alpha}$ & 0.000 & 0.005 & 0.005 & 0.010 & 0.000 & 0.270 & 0.420 & 0.410 & 0.160 \\
$89: 3$ & 0.020 & 0.010 & 0.005 & 0.010 & 0.200 & 0.250 & 0.010 & 0.250 & 0.230 \\
$89: 4$ & 0.780 & 0.590 & 0.625 & 0.815 & 0.370 & 0.280 & 0.070 & 0.210 & 0.470 \\
$90: 1$ & 0.200 & 0.375 & 0.365 & 0.160 & 0.070 & 0.050 & 0.070 & 0.230 & 0.040 \\
$90: 2$ & 0.000 & 0.005 & 0.000 & 0.000 & 0.070 & 0.080 & 0.040 & 0.220 & 0.030 \\
$90: 3^{\natural}$ & 0.645 & 0.660 & 0.700 & 0.660 & 0.320 & 0.360 & 0.820 & 0.300 & 0.550 \\
$90: 4$ & 0.005 & 0.010 & 0.030 & 0.015 & 0.280 & 0.380 & 0.040 & 0.250 & 0.210 \\
$91: 1$ & 0.000 & 0.005 & 0.000 & 0.003 & 0.230 & 0.050 & 0.130 & 0.240 & 0.020 \\
$91: 2$ & 0.000 & 0.000 & 0.000 & 0.000 & 0.170 & 0.060 & 0.000 & 0.250 & 0.000 \\
$91: 3^{\alpha}$ & 0.005 & 0.015 & 0.000 & 0.000 & 0.180 & 0.490 & 0.790 & 0.230 & 0.630 \\
$91: 4$ & 0.015 & 0.005 & 0.005 & 0.035 & 0.250 & 0.350 & 0.080 & 0.240 & 0.320 \\
\hline \hline
\end{tabular}

Notes: $A$ indicates that a downturn occured in output growth at that date 